\title{
An analytical fiber bundle model for pullout mechanics of root bundles
}

\author{
D. Cohen, ${ }^{1}$ M. Schwarz, ${ }^{1,2}$ and D. Or ${ }^{1}$
}

Received 21 September 2010; revised 28 April 2011; accepted 6 May 2011; published 19 August 2011.

[1] Roots in soil contribute to the mechanical stability of slopes. Estimation of root reinforcement is challenging because roots form complex biological networks whose geometrical and mechanical characteristics are difficult to characterize. Here we describe an analytical model that builds on simple root descriptors to estimate root reinforcement. Root bundles are modeled as bundles of heterogeneous fibers pulled along their long axes neglecting root-soil friction. Analytical expressions for the pullout force as a function of displacement are derived. The maximum pullout force and corresponding critical displacement are either derived analytically or computed numerically. Key model inputs are a root diameter distribution (uniform, Weibull, or lognormal) and three empirical power law relations describing tensile strength, elastic modulus, and length of roots as functions of root diameter. When a root bundle with root tips anchored in the soil matrix is pulled by a rigid plate, a unique parameter, $\kappa$, that depends only on the exponents of the power law relations, dictates the order in which roots of different diameters break. If $\kappa<1$, small roots break first; if $\kappa>1$, large roots break first. When $\kappa=1$, all fibers break simultaneously, and the maximum tensile force is simply the roots' mean force times the number of roots in the bundle. Based on measurements of root geometry and mechanical properties, the value of $\kappa$ is less than 1 , usually ranging between 0 and 0.7 . Thus, small roots always fail first. The model shows how geometrical and mechanical characteristics of roots and root diameter distribution affect the pullout force, its maximum and corresponding displacement. Comparing bundles of roots that have similar mean diameters, a bundle with a narrow variance in root diameter will result in a larger maximum force and a smaller displacement at maximum force than a bundle with a wide diameter distribution. Increasing the mean root diameter of a bundle without changing the distribution's shape increases both the maximum force and corresponding displacement. Estimates of the maximum pullout forces for bundles of 100 roots with identical diameter distribution for different species range from less than $1 \mathrm{kN}$ for barley (Hordeum vulgare) to almost $16 \mathrm{kN}$ for pistachio (Pistacia lentiscus). The model explains why a commonly used assumption that all roots break simultaneously overpredicts the maximum pullout force by a factor of about 1.6-2. This ratio may exceed 3 for diameter distributions that have a large number of small roots like the exponential distribution.

Citation: Cohen, D., M. Schwarz, and D. Or (2011), An analytical fiber bundle model for pullout mechanics of root bundles, J. Geophys. Res., 116, F03010, doi:10.1029/2010JF001886.

\section{Introduction and Background}

[2] By virtue of their tensile strength and extensive subsurface reach, roots contribute to the mechanical stabilization of steep slopes [e.g., Gray and Leiser, 1982]. Quantitative estimates of this effect is problematic because roots form complex heterogeneous biological networks whose geometry, extent, and mechanical characteristics evolve with time,

\footnotetext{
${ }^{1}$ Soil and Terrestrial Environmental Physics, ETH Zurich, Zurich, Switzerland.

${ }^{2}$ Swiss Federal Institute for Forest, Snow and Landscape Research, Birmensdorf, Switzerland.

Copyright 2011 by the American Geophysical Union. 0148-0227/11/2010JF001886
}

are difficult to measure in the field, and are challenging to model. In assessing the stability of a root-permeated slope, the prevailing approach has been to lump the effects of roots on soil strength into an apparent cohesion term added to the Mohr-Coulomb failure criteria [e.g., Waldron, 1977; Wu et al., 1979]

$$
\tau=c^{\prime}+\sigma^{\prime} \tan \phi^{\prime}+S
$$

where $\tau$ and $\sigma^{\prime}$ are the shear and effective normal stresses acting on a failure surface, $\phi^{\prime}$ is the effective angle of soil internal friction, $c^{\prime}$ is the effective soil cohesion, and $S$ is the shear resistance due to roots crossing that failure surface. Notwithstanding the fact that roots are anisotropic by nature and hence cannot be considered as true cohesion [Mitchell, 


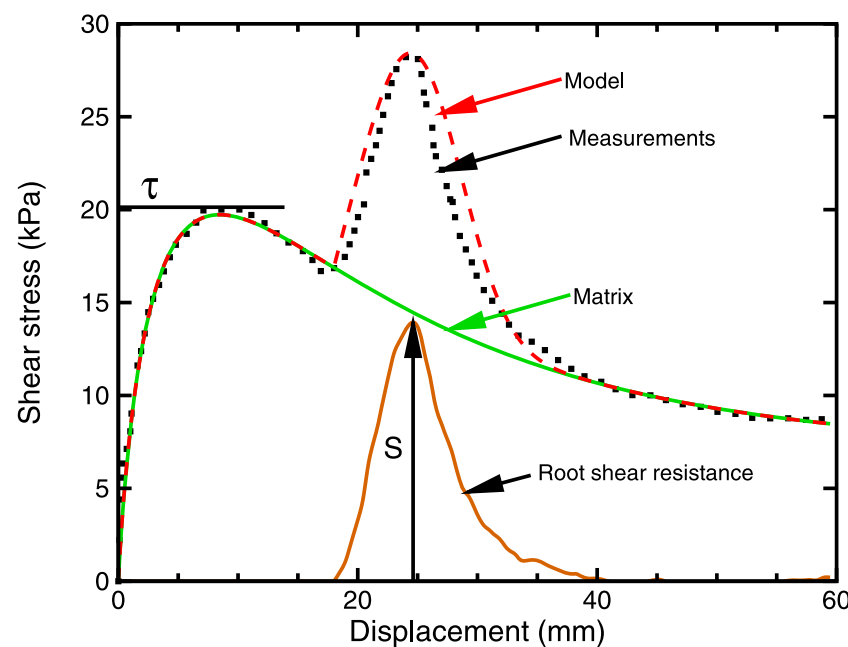

Figure 1. Example of an in situ shear test by Fannin et al. [2005] illustrating the effects of roots on the stress-strain behavior of soil. The measured stress-strain curve (black squares) shows two separate peaks due to matrix shear $(\tau)$ and roots tensile $(S)$ resistance. The green curve indicates shear stress due to the matrix only while the brown curve shows the effect of roots. The dashed red curve is the model result (see section 3.5).

2005], and despite observations that do not support that soils acquire additional cohesion from fibrous inclusions [e.g., Gray and Ohashi, 1983; Zornberg, 2002], this apparent root cohesion formulation has the practical advantage of distinguishing the strength of roots from that of the soil matrix in the same way that tensile strength of fiber-reinforced materials is computed as the sum of the yield strength of the matrix plus the tensional resistance of the fibers [e.g., Curtin and Takeda, 1998].

[3] The drawback of this approach, however, is that it blurs the distinction between the failure criteria (i.e., the Mohr-Coulomb rule) and the constitutive model of a rooted soil. Such a constitutive relation is the description needed to predict the strength increase of soils due to the presence of roots during deformation [e.g., Michalowski and Zhao, 1996; Ekanayake and Phillips, 1999; Sadek et al., 2010]. To illustrate this point, consider the in situ shear test by Fannin et al. [2005] of a cohesionless colluvium (see Figure 1, black squares) where the shear stress peaks twice, once due to soil mobilized at its peak shear strength and a second time due to the tensile strength mobilization of roots present in the sample. Note that these peaks occur at different displacements and thus simply adding root shear resistance $(S)$ to the shear strength of the matrix $(\tau)$ would grossly overestimate the resistance of the mixture to shear deformation and hence its resistance to mobilization in a landslide. For lack of a better and simpler approach, estimates of root-permeated soil shear strength [e.g., Riestenberg and Sovonick-Dunford, 1983; Zhou et al., 1998; Fan and Su, 2008; Mickovski et al., 2009] have for decades used the apparent root cohesion model first pioneered by Waldron [1977] and Wu et al. [1979] to estimate the term $S$ in equation (1). We briefly review these models to bring our own analysis into context.
[4] Early models assumed that roots are elastic fibers aligned perpendicular to a shear surface. Tensile root elongation across the interface adds shear resistance to the soil while also increasing the normal stress component on the shear surface. The term $S$ (expressed as a stress) can thus be written as the product of three terms:

$$
S=\Omega T_{M} \frac{A_{R}}{A_{S}} .
$$

The first term, $\Omega$, arises from the inclination of the root at an angle $\beta$ in the shear zone of thickness $Z$ and is

$$
\Omega=\sin \beta+\cos \beta \tan \phi^{\prime},
$$

where $\beta$ and $Z$ are related through the shear displacement $x$ by

$$
\tan \beta=\frac{x}{Z}
$$

The second term, $T_{M}$, is the mobilized strength of roots (here in units of stress). The third term, $A_{R} / A_{S}$, is the ratio of the cross-sectional area of roots cutting the shear plane over the area of the shear plane considered (this ratio is commonly called the root area ratio or RAR for short, a dimensionless quantity). Assuming a limited range of $\beta$ (between 40 and 70 degrees) and reasonable values of $\phi^{\prime}, \Omega$ is of order 1 , typically ranging between 0.6 and 1.3 . For roots not perpendicular to the shear surface this range can extend downward to slightly negative values [see Thomas and Pollen-Bankhead, 2010, for a detailed discussion]. Note that equation (2) states that $S$ depends on displacement through $\beta$ and is linearly proportional to the RAR but is not sensitive to the actual distribution of root sizes, an important factor that will be discussed below. Where these early models differ is in the estimation of $T_{M}$.

[5] Waldron [1977] balanced root-soil friction and tension in the root during shearing of a soil layer to obtain (with several ancillary assumptions) the first model that exposed a displacement dependence of tension in the root:

$$
T_{M}=\left(\frac{4 \tau^{\prime} Z E}{\phi}\right)^{1 / 2}(\sec \beta-1)^{1 / 2},
$$

where $\tau^{\prime}$ is root-soil friction, and $E$ and $\phi$ are the root elastic modulus and diameter, respectively (recall that $\beta$ is a function of $x$ ). The elastic modulus $E$ arises from Hooke's law when equating one half of the tension in the root below the shear plane (the average tension assuming tension decreases linearly with depth below the shear plane) to the strain, i.e.,

$$
\frac{T_{M}}{2}=\frac{\Delta l}{l} E
$$

where $l$ is the initial length of the root (above and below the shear plane) over which tension is active, and $\Delta l$ the change in root length due to shearing. The latter term is

$$
\Delta l=Z(\sec \beta-1),
$$

while $l$ is obtained from the tension in the root due to rootsoil friction. Assuming $l$ changes little during shearing

$$
l=\frac{T_{M} \phi}{2 \tau^{\prime}} .
$$


Combining the last 3 equations yields equation (5). Waldron [1977] assumed all roots had identical diameters and properties. Thus, for $N$ roots, each one of cross-sectional area $A=\pi \phi^{2} / 4, S$ can be written as (using root area $A$ instead of root diameter $\phi$ )

$$
S^{\text {Waldron }}=\Omega \delta E^{1 / 2} \frac{N A^{3 / 4}}{A_{S}},
$$

where

$$
\delta=\left(2 \pi^{1 / 2} \tau^{\prime} Z\right)^{1 / 2}(\sec \beta-1)^{1 / 2},
$$

and $\Omega$ is given by equation (3). Noting that since $N A=A_{R}$, root shear resistance $S$ in Waldron's model is inversely proportional to $A^{1 / 4}$ or, equivalently, to $\phi^{1 / 2}$. This means that small roots will provide more shear resistance than larger roots for an equivalent RAR. In Waldron's [1977] model, $T_{M}$ can be less than the critical, breaking, strength of the root if $l$ exceeds the root length, $L$, in which case the root slips out of the soil. Waldron's [1977] model is the first to include the concept of an activated root length $(l)$. Waldron [1977], and later Waldron and Dakessian [1981], carefully discussed cases when roots either slip or break depending on that activated length (for further details, see also Gray and Sotir [1996]). Using the concept of activated root length, Waldron and Dakessian [1981] explicitly considered the progressive failure of roots during deformation. Waldron's [1977] physical model has the disadvantage that some of the key parameters such as $Z, L$, and $\beta$ are not known or difficult to estimate.

[6] Waldron and Dakessian [1981] (WD) extended Waldron's [1977] analysis to roots of different diameter classes and obtained (again using area instead of root diameter)

$$
S^{\mathrm{WD}}=\Omega \delta \frac{1}{A_{S}} \sum_{i=1}^{m} n_{i} E_{i}^{1 / 2} A_{i}^{3 / 4}
$$

where the summation index $i$ is over the number of root diameter classes $m, E_{i}$ and $A_{i}$ are the elastic modulus and the cross-sectional area, respectively, of roots in class size $i, n_{i}$ is the number of roots in class size $i$, and $\sum_{i=1}^{m} n_{i}=N$. Many of the practical models use averages of diameters (or areas) and strengths in various combinations [Gray and Sotir, 1996]. This approach could also be used to express equation (11) as

$$
S^{\mathrm{WD}}=\Omega \delta \frac{m}{A_{S}}\left\langle n E^{1 / 2} A^{3 / 4}\right\rangle
$$

where the \langle\rangle denotes an expected value, i.e.,

$$
\left\langle n E^{1 / 2} A^{3 / 4}\right\rangle=\frac{1}{m} \sum_{i=1}^{m} n_{i} E_{i}^{1 / 2} A_{i}^{3 / 4}
$$

[7] Independent of the work of Waldron [1977], Wu et al. [1979] presented a different and simpler root-reinforcement model assuming that stresses in all roots crossing a shear surface are simultaneously at their critical tensile strength (and thus all roots are expected to fail simultaneously). With this hypothesis the resulting shear resistance is

$$
S^{\mathrm{Wu}}=\Omega \frac{1}{A_{S}} \sum_{i=1}^{m} n_{i} T_{i} A_{i},
$$

where $T_{i}$ is the root strength (expressed as stress) at which roots of class size $i$ and cross-sectional area $A_{i}$ break. Using averages, this equation can be written as

$$
S^{\mathrm{Wu}}=\Omega \frac{m}{A_{S}}\langle n T A\rangle=\Omega \frac{m}{A_{S}}\langle n F\rangle,
$$

where $F$ is a measurable force threshold at which roots break $(F \equiv T A)$. An attempt to further simplify equation (15) was, to the best of our knowledge, first mentioned by Gray and Leiser [1982] (GL) and assumed that the average of the product of root strength $T$ and root area $A$ could be replaced by the average root strength times the total area of the roots, i.e.,

$$
S^{\mathrm{GL}}=\Omega \frac{m^{2}}{A_{S}}\langle n T\rangle\langle n A\rangle,
$$

or

$$
S^{\mathrm{GL}}=\Omega m\langle n T\rangle \frac{A_{R}}{A_{S}} .
$$

This simplification has the significant advantage that it requires knowledge of only the $\operatorname{RAR}\left(A_{R} / A_{S}\right)$ and the average root strength $(m\langle n T\rangle$, in terms of stress). Unfortunately, this is a worse approximation than $W u$ et al. [1979] model because it overestimates the actual root reinforcement (expressed as soil shear resistance $S$ ) since the mathematical operator $m^{2}\langle n T\rangle\langle n A\rangle$ is much larger than $m\langle n T A\rangle$. Moreover, Equation (17) has often been incorrectly associated with the models of Wu et al. [1979] or Waldron [1977].

[8] The assumptions made by $W u$ et al. [1979] and the later simplification of Gray and Leiser [1982] imply that the peak tensile strength of roots is (1) additive, (2) independent of displacement (and thus fully available at all displacements), and (3) that all roots break simultaneously. This implies that $W u$ et al. [1979] model overpredicts values of root reinforcement and hence can potentially underestimate a slope's susceptibility to failure [e.g., Montgomery et al., 2009; Schwarz et al., 2010b]. These assumptions are also in conflict with numerous field and laboratory observations [e.g., Pollen et al., 2004; Docker and Hubble, 2008; Mickovski et al., 2009; Loades et al., 2010]. Recently, Pollen and Simon [2005], reintroduced the notion of progressive failure implicit in the early work of Waldron [1977] and proposed the fiber bundle model (FBM) [Daniels, 1945; Herrmann and Roux, 1990; Cohen et al., 2009] to simulate roots in soils as a bundle of fibers that rupture progressively under an increasing load. Since then, Pollen and Simon's [2005] model and subsequent formulations [Pollen, 2007; Pollen-Bankhead and Simon, 2009; Thomas and Pollen-Bankhead, 2010] have been used extensively to compute root reinforcement (the term $T_{M}$ in equation (2)) [e.g., Bischetti et al., 2005; Hales et al., 2009; Mickovski et al., 2009]. The models of Pollen and Simon [2005], Pollen [2007], Pollen-Bankhead and Simon [2009], and Thomas 
and Pollen-Bankhead [2010], however, do not take into account the combined effects of root diameter distribution, geometry (length and diameter), and mechanics (tensile strength and elastic modulus) in a unified root-reinforcement model. Also, because in these models bundle deformation is controlled by load instead of displacement, the root bundle force-displacement curve (i.e., the constitutive relation for root bundles or pullout curve) is not available past the maximum force, precluding estimates of the residual tensile force.

[9] To remedy some of these issues and to extend the analyses to include lateral root reinforcement, Schwarz et al. [2010a] proposed the Root Bundle Model (RBM) for estimating lateral root reinforcement. Focusing on the mechanical role of lateral roots meant that $S$ could be directly linked to $T_{M} \times A_{R} / A_{S}$ without the need for a local correction factor linked to root orientation (i.e., $\Omega=1$ ) since lateral roots pulled out from a vertical soil profile can be considered, to a first approximation, as oriented parallel to the direction of pull [see also Riestenberg and SovonickDunford, 1983]. In contrast with the models of Pollen and Simon [2005], Pollen [2007], Pollen-Bankhead and Simon [2009], and Thomas and Pollen-Bankhead [2010], the RBM is a displacement-controlled fiber bundle model that takes into account root strength, elastic modulus, and length as functions of diameter. The variation of root diameter along root length, root tortuosity, branching points and their frequency, soil moisture, and root-soil friction are also explicitly considered in the RBM. By imposing displacement rather than load, the RBM avoids ambiguities associated with load redistribution rules and yields the complete force-displacement curve including root's residual force beyond the maximum force. However, the central feature of the RBM is the evaluation of the cumulative progressive mobilization of root strength along the activation length during pullout due to root-soil friction for each root in the bundle, an element present in the early model of Waldron [1977] but omitted in the model of Wu et al. [1979] and subsequent FBMs. Unlike Waldron [1977], however, no assumptions are made in the RBM regarding the tensile force distribution along the activated length of the root. Owing to feedbacks, the RBM evaluation of root tension and activation length, which helps determine whether roots slip our of the soil or break, necessitates a numerical implementation. The RBM was tested using in situ field pullout tests and laboratory measurements [Schwarz et al., 2011] of Norway spruce roots. Schwarz et al. [2010a] noted that some model parameters such as effective soil pressure and water content had negligible effect on the maximum pullout force and corresponding displacement relative to other parameters. They emphasized that most of the variance in their results could be attributed to rootdiameter distribution and to geometrical characteristics such as root length.

[10] Based on these findings, we propose a simpler model, the Analytical Fiber Bundle Model (AFBM), stripped of much of the complexity of the model of Schwarz et al. [2010a], yet retaining the salient features of a standard fiber bundle for lateral root reinforcement. The new model is augmented by three empirical equations that describe root tensile strength (or force), elastic modulus, and length as functions of root diameter. The model assumes that roots are well anchored into the soil and hence break instead of slip out in accord with many field observations of landslide scarps [e.g., Wu et al., 1979; Abe and Ziemer, 1991; Schmidt et al., 2001]. With these assumptions, analytical formulas for the constitutive force-displacement curve (pullout curve, $P(x)$ ) of bundles of roots described by uniform, Weibull, or lognormal root-diameter distribution can be obtained. The maximum force, $P_{\max }$, a root bundle can sustain is related to lateral root reinforcement $S$ by

$$
S=\frac{P_{\max }}{A_{S}} .
$$

[11] The objectives of this work were to: (1) present the new model and derive analytical expressions for $P(x)$ and $P_{\max } ;(2)$ explore model parameters and their effects on pullout curves; and (3) relate earlier models of root reinforcement to the new model. Although the model naturally focuses on lateral root reinforcement, it applies equally well to the shear resistance of roots crossing a shear plane by reintroducing the factor $\Omega$ (equation (2)) into equation (18) although we believe this step unnecessary since, to a first approximation, $\Omega$ is of order 1 . The model could also have wider engineering applications for situations where a fibrous material is used as reinforcement and is pulled out of a cracked matrix, like in desiccation cracking of soils reinforced with fibers [e.g., Rifai and Miller, 2009], fiberreinforced soils or sands [e.g., Gray and Ohashi, 1983; Sadek et al., 2010; Silva dos Santos et al., 2010], and manmade fiber-reinforced materials such as concretes and composites [e.g., Curtin and Takeda, 1998].

\section{Model Description}

[12] To estimate the pullout force of root bundles as a function of displacement, we model roots in soil as straight, parallel, elastic-brittle fibers (Figure 2). Each fiber has a diameter, $\phi$, constant along its length. Diameters are drawn randomly from a probability density function $p_{\phi}(\phi)$. Fibers are anchored in the ground at their tips and pulled at their bases, parallel to their long axes, by controlling displacement (Figure 2b). Each fiber breaks at some critical displacement that depends on the fiber geometry and its mechanical properties. Once broken, fibers can either (1) support no load or (2) hold a residual load due to fiber-matrix friction that is proportional to the length of the fiber that is still being pulled. We assume no interactions between neighboring fibers. Consistent with observations and estimations of minimal reduction of diameter during pullout [Ennos, 1990; Schwarz et al., 2010a] fiber diameter is assumed to remain constant.

\subsection{Geometrical and Mechanical Characteristics}

[13] We assume that each fiber has the following geometrical and mechanical characteristics:

$$
\begin{aligned}
\frac{T}{T_{o}} & =\left(\frac{\phi}{\phi_{o}}\right)^{-\alpha}, \\
\frac{E}{E_{o}} & =\left(\frac{\phi}{\phi_{o}}\right)^{-\beta}, \\
\frac{L}{L_{o}} & =\left(\frac{\phi}{\phi_{o}}\right)^{\gamma},
\end{aligned}
$$




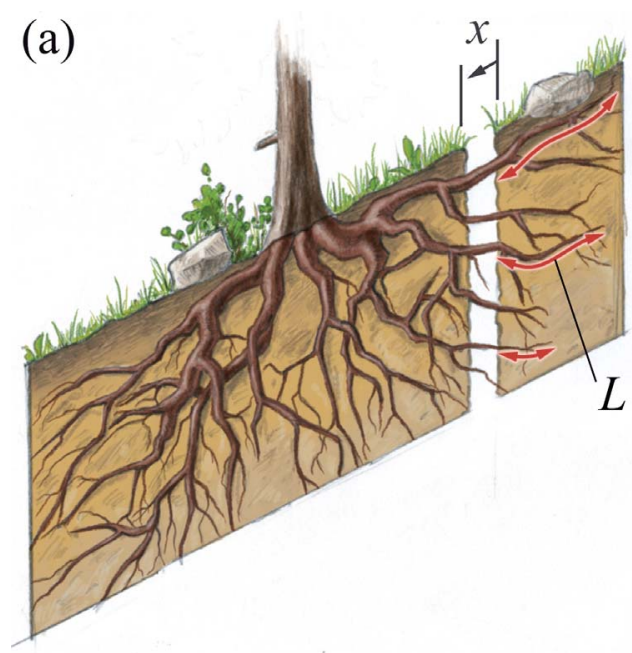

(b)

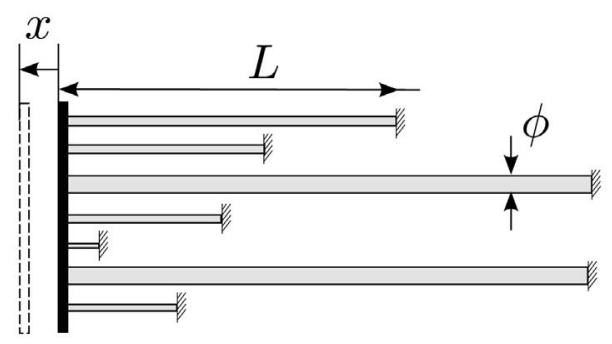

Figure 2. (a) Lateral pullout of roots on a slope across a tension crack upstream of a tree showing root anchoring length $L$ and displacement $x$. (b) Idealized bundle of roots. Roots are straight fibers of length $L$ and diameter $\phi$ aligned parallel to each other, anchored at their tips (right) and pulled at their bases (left) parallel to their axes by a rigid plate.

where $T$ is the threshold tensile strength, $E$ is the elastic (Young's) modulus, $L$ is the length, $T_{o}, E_{o}$, and $L_{o}$, are characteristic tensile strength, modulus, and length, respectively, $\phi_{o}$ is a reference diameter (so that $\phi / \phi_{o}$ is dimensionless), and $\alpha, \beta$, and $\gamma$ are power law exponents. The negative sign in front of $\alpha$ and $\beta$ is used because strength and elastic modulus are usually inversely related to diameter (restrictions on the value of these exponents will be discussed later). For roots, the fiber length, $L$, represents the distance to the point of anchoring (see Figure 2a). It is equivalent to the minimum length required to load the root to failure in the model of Waldron [1977]. Note, however, that here $L$ is a property of the root geometry and not the outcome of a force balance between root-soil friction and tensile stress.

[14] These empirical relationships are the most commonly used to describe root characteristics [e.g., Waldron and Dakessian, 1981; Gray and Sotir, 1996; Abernathy and Rutherfurd, 2001; Pollen and Simon, 2005; Tosi, 2007; Bischetti et al., 2009; Hales et al., 2009; Preti and Giadrossich, 2009; Schwarz et al., 2010a]. Other relationships, such as exponentials or quadratics, have also been used to relate threshold strength, force, or elastic modulus to diameter [e.g., Zhou et al., 1998; Operstein and Frydman, 2000; Tosi, 2007; Hales et al., 2009]. All of these relationships are best fits to data of individual roots that, not surprisingly, display rather large variations and where the coefficient of determination $\left(r^{2}\right)$ can be as low as 0.3 to 0.5 [e.g., Tosi, 2007; Bischetti et al., 2009; Preti and Giadrossich, 2009]. Note that these power law empirical equations could also describe other fibrous materials such as animal and vegetal fibers like wool, jute, flax, or hemp [e.g., O'Connell and Yeiser, 1954; Charlet et al., 2007; Beckermann and Pickering, 2009; Virk et al., 2009] or man-made fibers like glass, graphite, or carbon [e.g., Otto, 1955; Jones and Duncan, 1971; Lee et al., 2007] and thus be used for estimating the strength of bundles of fibers of such material embedded in a matrix that has begun to fail and hence carries negligible tensional load.

[15] Equation (19) can be replaced by a threshold force versus diameter relationship

$$
\frac{F}{F_{o}}=\left(\frac{\phi}{\phi_{o}}\right)^{\lambda},
$$

where $F$ is the threshold tensile force required to break the fiber, $\lambda=2-\alpha$, and $F_{o}=\pi \phi_{o}^{2} T_{o} / 4$.

[16] Inverting the geometrical and mechanical characteristics and using equation (22), one can write diameter, elastic modulus, and length as functions of threshold force, thus linking our model to standard fiber bundle model analyses where force (and not diameter) is the fundamental variable:

$$
\begin{gathered}
\phi=\phi_{o}\left(\frac{F}{F_{o}}\right)^{1 / \lambda}, \\
E=E_{o}\left(\frac{F}{F_{o}}\right)^{-\beta / \lambda}, \\
L=L_{o}\left(\frac{F}{F_{o}}\right)^{\gamma / \lambda} .
\end{gathered}
$$

\subsection{Force in a Single Fiber}

[17] Since each fiber is linear elastic, the tensional force, $f$, in a fiber at displacement $x$ (see Figure 2b) is given by

$$
\begin{aligned}
& f=\frac{E A}{L} x, \quad x<x_{c}, \\
& f=0, \quad x \geq x_{c},
\end{aligned}
$$

where $A=\pi \phi^{2} / 4$ is the fiber's cross-sectional area and $x_{c}$ is the displacement at which the fiber breaks. Substituting equations (23), (24), and (25) into (26a) yields

$$
f=W_{o} F^{\kappa} x,
$$

where

$$
W_{o}=\frac{\pi}{4} \frac{E_{o} \phi_{o}^{2}}{L_{o} F_{o}^{\kappa}},
$$



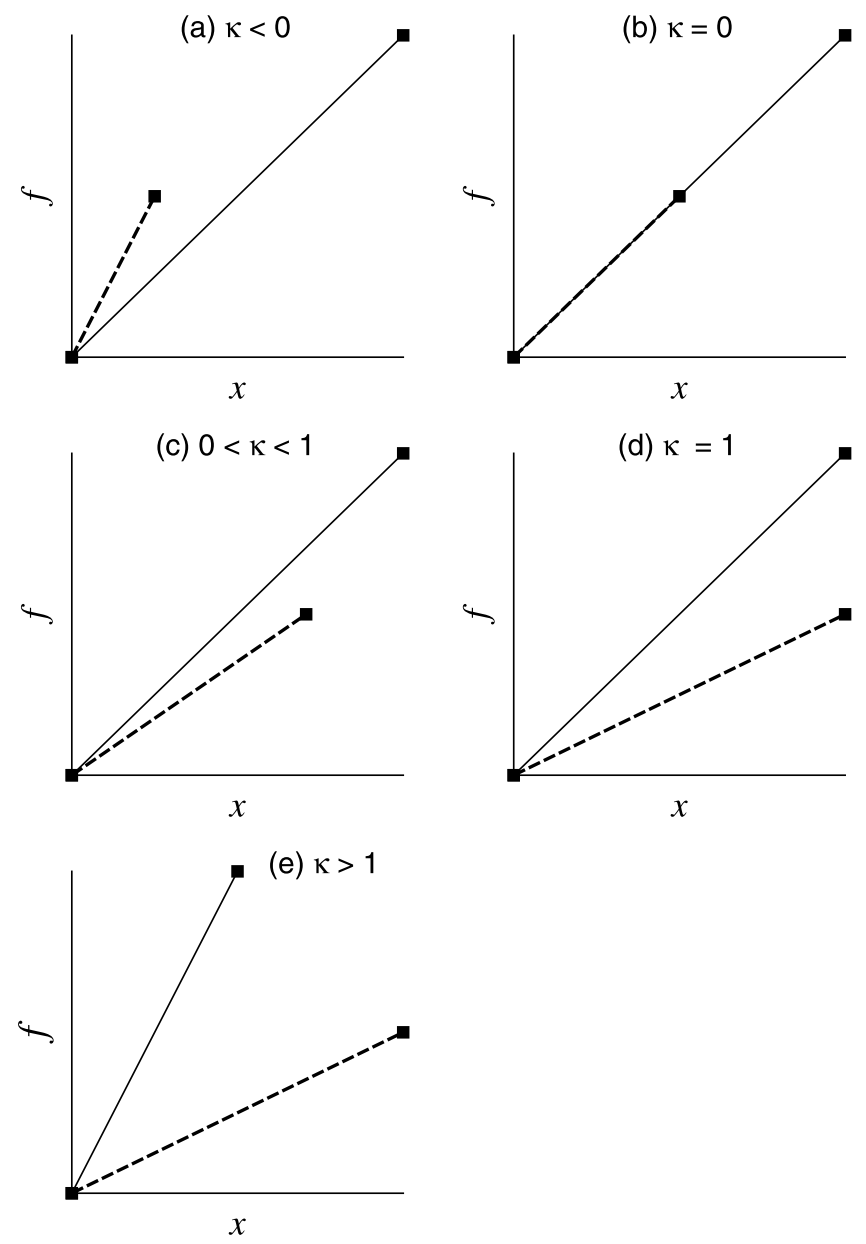

Figure 3. Force-displacement ( $f$ versus $x)$ elastic behavior of two fibers with two different threshold forces for different values of $\kappa$. The stronger fiber (having the larger threshold force) is shown with a solid line. (a) When $\kappa<0$, the weaker fiber holds more force but breaks first. (b) When $\kappa=0$, both fibers hold equal force and the weaker fiber breaks first. (c) When $0<\kappa<1$, the weaker fiber holds less force and breaks first. (d) When $\kappa=1$, both fibers break at the same displacement with the weaker fiber holding less force. (e) When $\kappa>1$, the strongest fiber breaks first and holds more force.

and

$$
\kappa=\frac{2-(\beta+\gamma)}{\lambda} .
$$

$W_{o}$ has units of $\left[\mathrm{M}^{1-\kappa} \mathrm{L}^{1-\kappa} \mathrm{T}^{2(\kappa-1)}\right]$, where $\mathrm{M}, \mathrm{L}$, and $\mathrm{T}$ are, respectively, mass, length, and time. Using equation (28) and the relationship between $F_{o}$ and $T_{o}$, a dimensionless form of equation (27) is

$$
f^{\star}=W_{o}^{\star} F^{\star^{\kappa}} x^{\star}
$$

where $f^{\star}=f / F_{o}, F^{\star}=F / F_{o}, x^{\star}=x / L_{o}$, and $W_{o}^{\star}=E_{o} / T_{o}$.

[18] The displacement at failure $\left(x_{c}\right)$ is obtained by setting $f=F$ in the left-hand side of equation (27):

$$
x_{c}=\frac{F^{1-\kappa}}{W_{o}} .
$$

The order in which fibers of different threshold forces break depends on the sign of the parameter $1-\kappa$. Also, since the force in a single fiber is linearly proportional to displacement, at a given displacement $x$, the fiber that holds a higher tensional force depends on the sign of $\kappa$ (see equation (27)). The five possible combinations of tensional forces in and time of failure of two fibers of different diameters are shown in Figure 3. Note that smaller fibers do not necessarily hold less force (cases $\kappa \leq 0$ ).

\subsection{Probability Density Functions}

[19] We now assume that the probability density function for root diameter, $p_{\phi}(\phi)$, is either a uniform distribution,

$$
p_{\phi}^{\mathrm{U}}\left(\phi ; \phi_{a}, \phi_{b}\right)=\frac{1}{\phi_{b}-\phi_{a}}, \quad \phi_{a} \leq \phi \leq \phi_{b},
$$

a Weibull distribution,

$$
p_{\phi}^{\mathrm{W}}\left(\phi ; m_{\phi}, k_{\phi}\right)=\frac{m_{\phi}}{k_{\phi}}\left(\frac{\phi}{k_{\phi}}\right)^{m_{\phi}-1} \exp \left[-\left(\frac{\phi}{k_{\phi}}\right)^{m_{\phi}}\right],
$$

where $m_{\phi}$ and $k_{\phi}$ are the shape and scale factors, respectively, or a lognormal distribution

$$
p_{\phi}^{\mathrm{L}}\left(\phi ; \mu_{\phi}, \sigma_{\phi}\right)=\frac{1}{\sqrt{2 \pi} \sigma_{\phi} \phi} \exp \left[-\frac{\left(\ln \phi-\mu_{\phi}\right)^{2}}{2 \sigma_{\phi}^{2}}\right],
$$

where $\mu_{\phi}$ and $\sigma_{\phi}$ are the mean and standard deviation of $\ln \phi$, respectively. The latter two distributions are commonly used to analyze root diameter distribution and often give better fits [e.g., Scanlan and Hinz, 2010]; the simple form of the uniform distribution yields analytical solutions for the maximum pullout force (see section 2.5 below).

\subsection{Bundle Pullout Force}

[20] In our model, all fibers in the bundle are deformed by an identical displacement. We choose this approach (as opposed to increasing stress) because: (1) most physical laboratory and field experiments are done by imposing a constant shear strain rate to either a rooted soil mass or a constant tensile strain rate to an individual root or root bundle, (2) we avoid the issue of load redistribution from the broken to the surviving fibers knowing that some of these rules yield nonidentical displacement for the surviving fibers, a response that matches no experimental method, (3) only displacement-control models can evaluate the forcedisplacement behavior of the bundle past the maximum force, and (4) only displacement-control models yield manageable analytical solutions when the geometry and the mechanical properties of the fibers are nonuniform.

[21] By imposing a displacement, fibers in the bundle break one at a time with increasing displacement without any load redistribution between fibers and the bundle pullout force, $P(x)$, can be obtained directly by integration of equation (27) over the fiber threshold force distribution

$$
P(x)=N W_{o} x \int_{F_{L}}^{F_{U}} F^{\kappa} p_{F}(F) d F,
$$

where $N$ is the total number of fibers in the bundle, $F_{L}$ and $F_{U}$ are lower and upper limits of integration spanning intact 
Table 1. Force Threshold Probability Density Functions, $p_{F}(F)$, for Different Root Diameter Probability Density Functions, $p_{\phi}(\phi)^{\mathrm{a}}$

\begin{tabular}{ccc}
\hline$p_{\phi}(\phi)$ & $p_{F}(F)$ & Parameters \\
\hline Uniform & $\frac{F^{\frac{1-\lambda}{\lambda}}}{\lambda\left(F_{b}^{1 / \lambda}-F_{a}^{1 / \lambda}\right)}$ & $F_{a}=F_{o}\left(\frac{\phi_{a}}{\phi_{o}}\right)^{\lambda}$, \\
& $F_{b}=F_{o}\left(\frac{\phi_{b}}{\phi_{o}}\right)^{\lambda}$, \\
& $\langle F\rangle=\frac{1}{1+\lambda} \frac{\frac{1+\lambda}{F_{b}^{\lambda}}-F_{a}^{\frac{1+\lambda}{\lambda}}}{F_{b}^{1 / \lambda}-F_{a}^{1 / \lambda}}$ \\
Weibull & $\frac{m_{F}}{k_{F}}\left(\frac{F}{k_{F}}\right)^{m_{F}-1} \exp \left[-\left(\frac{F}{k_{F}}\right)^{m_{F}}\right]$ & $m_{F}=m_{\phi} / \lambda$, \\
& & $k_{F}=F_{o}\left(k_{\phi} / \phi_{o}\right)^{\lambda}$, \\
Lognormal & $\frac{1}{\sqrt{2 \pi} \sigma F^{F}} \exp \left[-\frac{\left(\ln F-\mu_{F}\right)^{2}}{2 \sigma_{F}^{2}}\right]$ & $\mu_{F}=\ln F_{o}-\lambda \ln \phi_{o}+\mu \phi \lambda$, \\
& & $\sigma_{F}=\lambda \sigma \phi$, \\
& & $\langle F\rangle=\exp \left(\frac{\sigma_{F}^{2}}{2}+\mu_{F}\right)$ \\
\hline
\end{tabular}

${ }^{\mathrm{a}}$ The mean force, $\langle F\rangle$, is also given.

fibers, and $p_{F}(F)$ is the probability density function expressed in terms of force. Since the integration is over $F$, $p_{F}(F)$ must be obtained from $p_{\phi}(\phi)$ by a change of variable using equation (22). Since $F$ is a monotonically increasing function of $\phi$ (i.e., $\lambda>0$, see section 4.1), Weibull and lognormal probability density functions do not change form under the transformation (parameters are modified, however) and the uniform probability density function is transformed into a power law distribution over a finite range of force. Equations for $p_{F}(F)$ are given in Table 1 .

[22] To find the limits of integration of equation (35), it is useful to plot equation (31) for different values of $1-\kappa$ (Figure 4). If $\kappa=1$, all fibers break at once when the displacement $x$ reaches the value of $x_{c}=1 / W_{o}$ independent of their threshold forces (see also Figure 3d). If $\kappa<1$, with increasing displacement, fibers break in increasing order of

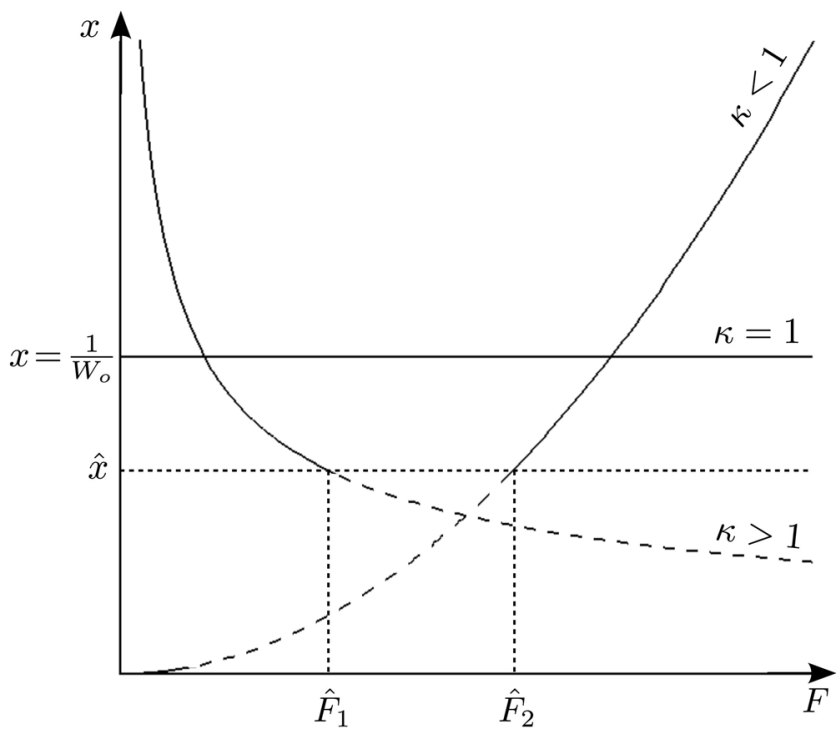

Figure 4. Fiber threshold force-displacement maps for different values of $\kappa$. See text for explanation. their threshold force: at a displacement $x=\hat{x}$ (see Figure 4), fibers with threshold forces $F \leq \hat{F}_{2}$ (dashed part of $\kappa<1$ curve below $x=\hat{x}$ ) are broken. The opposite is true if $\kappa>1$ : fibers with threshold forces $F \geq \hat{F}_{1}$ (dashed part of $\kappa<1$ curve below $x=\hat{x}$ ) are broken. Inverting equation (31) for $F$ yields the limits of integration (see Table 2 ).

[23] Since the threshold force increases monotonically with diameter (equation (22), $\lambda>0$ ), small diameter fibers break first when $\kappa<1$ and last when $\kappa>1$. This result is important: it arises as a consequence of (1) the power law exponents that control geometrical (length versus diameter) and mechanical (strength and modulus versus diameter) properties, and (2) the constraint that fibers undergo identical displacements (pull with a rigid plate).

[24] Had the bundle been stretched using a rigid plate by imposing a load (i.e., a force) instead of a displacement, load redistribution to stronger fibers is such a highly nonlinear process that an analytical formula for the pullout force cannot be derived except for the case of identical fibers [Sornette, 1989]. For identical fibers, global load redistribution rules (also called democratic load sharing) imply that each fiber holds the same force (also the same stress since fibers have the same diameters). For fibers with heterogeneous geometrical and mechanical properties such as those given by equations (19), (20), and (21), however, global (democratic) load sharing implies that the forces $f_{i}$ and $f_{j}$ in two fibers of different diameters $\phi_{i}$ and $\phi_{j}$, respectively, are not equal but are related by

$$
\frac{f_{i}}{f_{j}}=\left(\frac{\phi_{i}}{\phi_{j}}\right)^{2-\beta-\gamma} .
$$

If fibers have identical length and elastic modulus $(\beta=\gamma=0)$ but different diameters, then they support equal stresses (but not equal forces).

[25] Our model assumes that all roots undergo the same displacement. This homogeneous behavior may apply only

Table 2. Limits of Integration of Equation (35) for the Different Distribution Functions and as a Function of the Parameter $\kappa$ and the Displacement $x^{\mathrm{a}}$

\begin{tabular}{lcc}
\hline & Lower Limit & Upper Limit \\
\hline$X<\frac{1}{W_{o}}$ & All Distributions, $\kappa=1$ & \\
$X \geq \frac{1}{W_{o}}(P=0)$ & 0 & - \\
& - & \\
$x<\frac{F_{a}^{1-\kappa}}{W_{o}}$ & Uniform, $\kappa<1$ & $F_{b}$ \\
$\frac{F_{a}^{1-\kappa}}{W_{o}} \leq x<\frac{F_{b}^{1-\kappa}}{W_{o}}$ & $F_{a}$ & $F_{b}$ \\
$x \geq \frac{F_{b}^{1-\kappa}}{W_{o}}(P=0)$ & $\left(W_{o} x\right)^{1 /(1-\kappa)}$ & - \\
$x<\frac{F_{b}^{1-\kappa}}{W_{o}}$ & - & \\
$\frac{F_{b}^{1-\kappa}}{W_{o}} \leq x<\frac{F_{a}^{1-\kappa}}{W_{o}}$ & Uniform, $\kappa>1$ & $F_{b}$ \\
$x \geq \frac{F_{a}^{1-\kappa}}{W_{o}}(P=0)$ & $F_{a}$ & $\left(W_{o} x\right)^{1 /(1-\kappa)}$ \\
$\kappa<1$, all $x$ & $F_{a}$ & - \\
$\kappa>1$, all $x$ & - & $\left(W_{o} x\right)^{1 /(1-\kappa)}$ \\
\hline
\end{tabular}

${ }^{\mathrm{a}}$ For ranges of $x$ that yield $P=0$ (all fibers are broken), no limits are indicated. 
locally to root bundles that span distances that scale with the anchoring length $L$. At larger scales relevant to landslides, displacements of roots pulled across shear zones or tension cracks are likely inhomogeneous because of variations in stresses but also because of inhomogeneities in the soil. Without measurements or models to constrain displacement, the ideal case of a rigid pull is a simplification of reality but offers a physically sound theory applicable at the local scale.

[26] Analytical expressions for the pullout force $P(x)$ as a function of displacement are obtained by substituting the probability density function (equation (32), (33), or (34)) into equation (35) and integrating using the limits in Table 2. As an illustration, for the Weibull distribution with $\kappa<1$ one obtains

$$
P(x)=N W_{o} k_{F}^{\kappa} x \Gamma\left(\frac{\kappa}{m_{F}}+1, \frac{\left(W_{o} x\right)^{\frac{m_{F}}{1-\kappa}}}{k_{F}^{m_{F}}}\right),
$$

where $\Gamma(\cdot, \cdot)$ is the upper incomplete Gamma function that arises from the integration of an exponential function with a power law over a bounded interval. Results for other distributions and cases are shown in Table 3.

\subsection{Maximum Pullout Force and Corresponding Displacement}

[27] Displacement at maximum pullout force, $x_{\max }$, is obtained by finding the solution to the equation $d P(x) / d x=0$. Then, the maximum pullout force is $P_{\max }=P\left(x_{\max }\right)$. This procedure does not yield obvious analytical expressions for the Weibull and lognormal distributions when $\kappa \neq 1$. In these cases, we show analytical expressions for the equation $d P(x) / d x=0$. Results for all distributions are given in Table 4 and detailed below.

[28] For the uniform distribution and for $\kappa<1$ the maximum force is reached inside the interval $F_{a}^{1-\kappa} / W_{o}<$ $x<F_{b}^{1-\kappa} / W_{o}$ while for $\kappa \geq 1$ the maximum force is obtained precisely at $x=F_{b}^{1-\kappa} / W_{o}$.

[29] For the Weibull and lognormal distributions, the displacement at maximum force can be obtained by solving an equation involving the incomplete Gamma function (Weibull distribution) or the error function (lognormal distribution). For the Weibull distribution and $\kappa<1$ we obtain the equation

$$
\Gamma(s, \eta)-\frac{m_{F}}{1-\kappa} \eta^{s} \mathrm{e}^{-\eta}=0,
$$

where $s=1+\kappa / m, \eta=A x^{m /(1-\kappa)}, A=W_{o}^{m /(1-\kappa)} / k_{F}^{m_{F}}$, and $\Gamma(\cdot, \cdot)$ is the upper incomplete Gamma function. Equation (38) can be solved for $\eta$ from which $x_{\max }$ can be calculated. The maximum pullout force can then be computed by substituting into the appropriate pullout force equation in Table 3. For the case $\kappa>1$ we get

$$
\gamma(s, \eta)+\frac{m_{F}}{1-\kappa} \eta^{s} \mathrm{e}^{-\eta}=0,
$$

where $\gamma(\cdot, \cdot)$ is the lower incomplete Gamma function. Equations for the lognormal cases are given in Table 4.

[30] As can be seen from equations in Table 4, the calculation of the maximum force depends only on four parameters: two that describe the threshold force distribution (either $F_{a}$ and $F_{b}$ (uniform), $m_{F}$ and $k_{F}$ (Weibull), or $\mu_{F}$ and $\sigma_{F}$ (lognormal)) 
Table 4. Analytical Expressions for the Maximum Pullout Force, $P_{\max }$, for the Three Distributions as a Function of the Parameter $\kappa^{\text {a }}$

\begin{tabular}{|c|c|c|c|}
\hline & $\kappa=1$ & $\kappa=-1 / \lambda$ & $\kappa<1$ \\
\hline Uniform & $\langle F\rangle$ & $\frac{\exp \left[\frac{1+\lambda}{\lambda} \ln F_{b}-1\right]}{(1+\lambda)\left(F_{b}^{1 / \lambda}-F_{a}^{1 / \lambda}\right)}$ & $\frac{\frac{1+\lambda}{F_{b}^{\lambda}}}{\left(F_{b}^{1 / \lambda}-F_{a}^{1 / \lambda}\right)} \frac{[\lambda(1-\kappa)]^{\frac{\lambda(1-\kappa)}{1+\kappa \lambda}}}{(1+\lambda)^{\frac{1+\lambda}{1+\kappa \lambda}}}$ \\
\hline Weibull & $\langle F\rangle$ & see $\kappa<1$ & $\Gamma(s, \eta)-\frac{m_{F}}{1-\kappa} \eta^{s} \mathrm{e}^{-\eta}=0$ \\
\hline Lognormal & $\langle F\rangle$ & see $\kappa<1$ & $\begin{array}{c}1+\operatorname{sgn}(1-\kappa) \operatorname{erf}(Y(x))-\operatorname{sgn}(1-\kappa) \frac{2 \exp \left(-Y^{2}(x)\right) \ln \left(W_{o} x\right)^{\kappa /(1-\kappa)}}{\sqrt{2 \pi} \sigma_{F}(1-\kappa)}=0 \\
\text { where } Y(x)=\frac{1}{\sqrt{2} \sigma}\left(\kappa \sigma_{F}^{2}+\mu_{F}+\ln \left(W_{o} x\right)^{1 /(1-\kappa)}\right)\end{array}$ \\
\hline
\end{tabular}

${ }^{\mathrm{a}}$ For the Weibull and lognormal distributions and for $\kappa \neq 1$, analytical expressions could not be derived and equations to obtain $x_{\max }$ are shown. See text for details. The symbol sgn means "sign of."

and two independent parameters that describe the combined geometrical and mechanical characteristics of the roots: $\lambda$ and $\kappa$ (uniform), or $W_{o}$ and $\kappa$ (Weibull, lognormal).

[31] Note that for $\kappa=1$, all distributions yield $P_{\max }=\langle F\rangle$, where $\langle F\rangle$ is the mean threshold force (see Table 2 for formulas). Thus, root reinforcement in the Mohr-Coulomb failure criteria ( $S$ in equation (1)) is $S=N\langle F\rangle / A_{S}$, a result equivalent to the model of $W u$ et al. [1979] (see equation (15)) without the factor $\Omega$ and with the average defined over the number of roots $N$ instead of the number of root class sizes $m$. In that special case the maximum force depends only on the force distribution parameters. Note that the force distribution parameters depend on the diameter distribution parameters and on equation (4), hence also on the parameters $F_{o}, \lambda$, and $\phi_{o}$.

\section{Model Evaluation}

[32] We are aware of only two publications on root reinforcement that provide a complete description of the geometry and mechanics of roots needed for testing our model: Waldron and Dakessian [1981] and Schwarz et al. [2010a]. Many studies do not provide key information such as diameter distribution, number, or elastic properties of roots [e.g., Zhou et al., 1998; Operstein and Frydman, 2000; Pollen and Simon, 2005; Fan and Su, 2008] making comparisons difficult and unreliable. Except for Waldron and Dakessian [1981] and Schwarz et al. [2010a], all neglect root length. Thus, we test our model by comparing it to their data which were obtained under different conditions and set ups: laboratory shear tests of soil reinforced with pine or barley roots [Waldron and Dakessian, 1981]; field pullout tests of Norway spruce roots [Schwarz et al., 2011]. These publications also provide comparisons of measurements with their own model against which our model can be evaluated. We also compare our model with the data of Mickovski et al. [2009], who provide detailed data of root-diameter distribution and comparisons with several other root-reinforcement models, and with the data of Fannin et al. [2005].

\subsection{Field Pullout Tests of Norway Spruce Roots}

[33] Schwarz et al. [2011] performed six field pullout tests of Norway spruce roots on vertical profiles dug at Uetliberg, Switzerland, totaling 36 roots. For our comparison we combine five of the six tests totaling 29 roots. Root diameters measured in these experiments were fitted to uniform, Weibull, and lognormal distributions to obtain the parameters $\phi_{a}, \phi_{b}$, $m_{\phi}, k_{\phi}, \mu_{\phi}$ and $\sigma_{\phi}$ (Figure 5 and Table 5). Root characteristics (parameters $\alpha, \beta, \gamma, T_{o}, E_{o}, L_{o}$ ) from Schwarz et al. [2010a] are given in Table 6 . Because natural roots are seldom perfectly straight, tortuosity affected the measured pullout force. This effect is not specifically included in the analytical model described here. However, it can be modeled by modifying the value of the characteristic elastic modulus $E_{o}$. Laboratory tensile stress tests [e.g., Commandeur and Pyles, 1991] indicate that roots can have an initially low stress-strain slope associated with a small elastic modulus due to tortuosity. In pullout tests of roots in soil [e.g., Mickovski et al., 2007], tortuosity reduces the stress-strain slope past a small displacement and causes roots to fail at higher displacements. To achieve the same displacement at failure as observed, an apparent elastic modulus of half the material modulus is used, a value close to the estimated secant modulus estimated by Commandeur and Pyles [1991].

[34] Figure 6 shows the pullout force as a function of displacement for the measurements of Schwarz et al. [2011], the analytical fiber bundle model, the RBM, and Wu et al. [1979] model. Results show that the AFBM captures both the maximum pullout force and its corresponding displacement. Unlike the RBM, individual root failure is not cap-

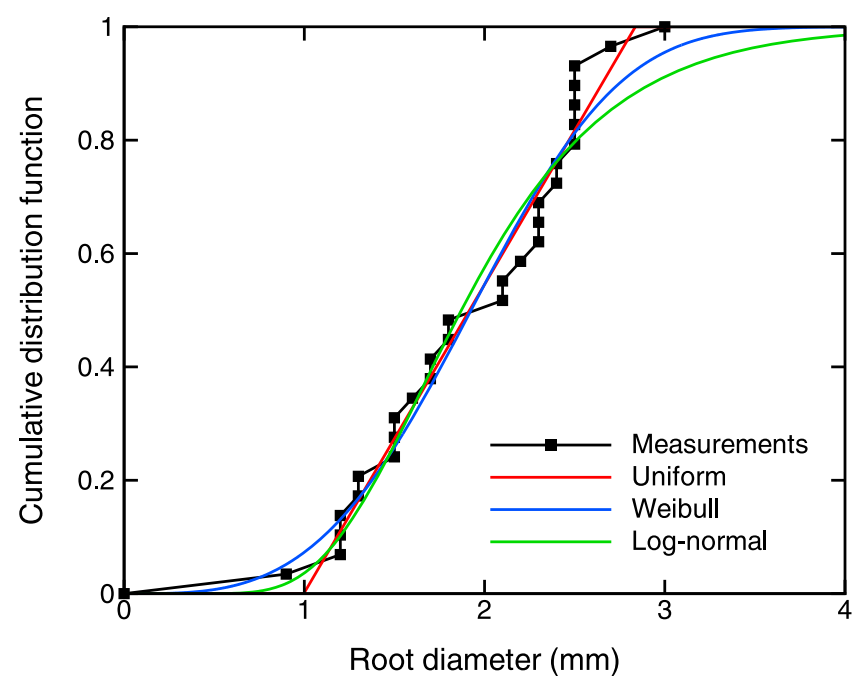

Figure 5. Measured root diameters [Schwarz et al., 2011] and fitted uniform, Weibull, and lognormal cumulative distributions. Parameters of the distributions are given in Table 5. 
Table 5. Distribution Parameters Used in Model Comparisons Based on Combining Five of Six Field Pullout Tests ${ }^{\mathrm{a}}$

\begin{tabular}{lcc}
\hline & Diameter & Force \\
\hline Uniform & $\phi_{a}=0.997 \mathrm{~mm}$ & $F_{a}=22.0 \mathrm{~N}$ \\
& $\phi_{b}=2.84 \mathrm{~mm}$ & $F_{b}=83.8 \mathrm{~N}$ \\
Weibull & $m_{\phi}=3.38$ & $m_{F}=2.64$ \\
& $k_{\phi}=2.15 \mathrm{~mm}$ & $k_{F}=58.7 \mathrm{~N}$ \\
Lognormal & $\mu_{\phi}=-6.28 \ln \mathrm{m}$ & $\mu_{F}=3.90 \operatorname{ln~N}$ \\
& $\sigma_{\phi}=0.349 \operatorname{ln~m}^{1 / 2}$ & $\sigma_{F}=0.447 \operatorname{ln~} \mathrm{N}^{1 / 2}$ \\
\hline
\end{tabular}

${ }^{\mathrm{a} S e e}$ Schwarz et al. [2011].

tured owing to the use of continuous distributions. Wu et al. [1979] model, by using the threshold (breaking) tensile stress of roots, overestimates the maximum force by about a factor of 2 and does not depend on displacement. Because Weibull and lognormal distributions include roots of all diameters $(0<\phi<\infty)$, the pullout forces at large displacement computed with the AFBM tapper off slowly owing to the residual forces held by the small proportion of large roots. The pullout force computed with the uniform distribution shows no such residual force because roots have finite sizes. Between the Weibull and lognormal distributions, the lognormal distribution carries a bigger fraction of large-diameter fibers and hence better fits the higher measured residual force. Although this tapering off mimics observations, in reality part of the residual pullout force is caused by friction between roots and soil during slippage of roots. This process can be included in our AFBM (see model description in Appendix A). Including it (Figure 6, dashed blue curve) results in a better fit with the measurements at large strain but the maximum pullout force is slightly overestimated owing to broken roots that now carry some pulling resistance from friction with the matrix.

\subsection{Shear of Barley-Reinforced Soil}

[35] Waldron and Dakessian [1981] performed several tests to measure barley root reinforcement of soils in shear.
They provided all necessary parameters of the power law equations needed for the model (see Table 6) and presented diameter distribution as charts of the numbers of roots belonging to a range of diameter (diameter class). We transformed these numbers to Weibull distribution parameters ( $m_{\phi}$ and $k_{\phi}$, see equation (33)) as follows: (1) we tabulated the data in terms of a continuous distribution function (cdf), (2) fitted the cdf to the Weibull distribution using nonlinear regression, (3) corrected the value of $k_{\phi}$ such that the mean of the Weibull distribution matched the mean root size indicated by Waldron and Dakessian [1981]. We obtained $m_{\phi}=3.0$ and $k_{\phi}=0.27 \mathrm{~mm}$.

[36] Figure 7 shows the data of Waldron and Dakessian [1981], their estimates using the model of Waldron [1977], and two estimates with our model: one with $\gamma$ (the power law exponent of root length) set to 0.5 as suggested by Waldron and Dakessian [1981] (this parameter was not measured explicitly by them), one with $\gamma=0.53$ which gives a better fit. The initial local maximum at a displacement of about $0.2 \mathrm{~cm}$ was attributed by Waldron and Dakessian [1981] to sample preparation and test procedures. With the latter value of $\gamma$, our model matches correctly the upward trend of root reinforcement with increasing displacement. That trend is not captured with the model of Waldron [1977]. Our model illustrates the rather large effect of changing the parameter $\gamma$ : a $6 \%$ difference results in a $28 \%$ increase in root reinforcement at a displacement of $1.5 \mathrm{~cm}$.

\subsection{Shear of Pine-Reinforced Soil}

[37] We follow the same procedure to compare Waldron and Dakessian [1981] shear test measurements of pineplanted soils. Power law exponents are given in Table 6 and we obtained $m_{\phi}=0.98$ and $k_{\phi}=1.5 \mathrm{~mm}$. Figure 8 shows the comparison between data, the model of Waldron [1977] and our model using two values of $\gamma, 1.0$ and 1.21. Our model underestimates significantly root reinforcement for $\gamma=1$ (value suggested by Waldron and Dakessian [1981]) but does well for $\gamma=1.21$ owing to the effect of $\gamma$ on the

Table 6. Geometrical and Mechanical Parameters of Roots ${ }^{\mathrm{a}}$

\begin{tabular}{|c|c|c|c|c|c|c|c|c|c|c|c|}
\hline Latin Name & $\begin{array}{l}\text { Common } \\
\text { Name }\end{array}$ & $\begin{array}{c}T_{o} \phi_{o}^{\alpha} \\
\left(\mathrm{MPa} \mathrm{mm}^{\alpha}\right)\end{array}$ & $\alpha$ & $\begin{array}{c}E_{o} \phi_{o}^{\beta} \\
\left.(\mathrm{MPa} \mathrm{mm})^{\beta}\right)\end{array}$ & $\beta$ & $\begin{array}{c}L_{o} \phi_{o}^{-\gamma} \\
\left(\mathrm{mm}^{1-\gamma}\right)\end{array}$ & $\gamma$ & $\begin{array}{c}F_{o} \phi_{o}^{-\lambda} \\
\left(\mathrm{N} \mathrm{mm}^{-\lambda}\right)\end{array}$ & $\lambda$ & $\left(\mathrm{MN}^{1-\kappa} \mathrm{mm}^{-1}\right)$ & $\kappa$ \\
\hline Picea abies $L .^{\mathrm{b}}$ & Spruce & 28.1 & 0.72 & 696 & 1. & 335 & 0.63 & 22.1 & 1.28 & 36.2 & 0.289 \\
\hline Hordeum vulgare ${ }^{\mathrm{c}}$ & Barley & 6.77 & 0.944 & 13.2 & 1.21 & 1581 & 0.5 & 5.32 & 1.28 & 0.185 & 0.275 \\
\hline Pinus Ponderosa ${ }^{\mathrm{c}}$ & Pine & 21.4 & 0.116 & 58.2 & 0.389 & 500 & 1. & 16.8 & 1.88 & 3.23 & 0.324 \\
\hline Medicago sativa ${ }^{\mathrm{d}}$ & Alfalfa & 29.4 & 0.780 & 714 & 1.12 & 335 & 0.63 & 23.1 & 1.220 & 15.2 & 0.207 \\
\hline Cistus $\mathrm{d}^{\mathrm{d}}$ & & 50.5 & 0.901 & 1090 & 1.11 & 335 & 0.63 & 1254 & 1.099 & 7.09 & 0.234 \\
\hline Pistacia lentiscus ${ }^{\mathrm{d}}$ & Pistachio & 69.4 & 0.474 & 362 & 1.16 & 335 & 0.63 & 54.5 & 1.526 & 3.24 & 0.136 \\
\hline Rosmarinus officinalis ${ }^{\mathrm{d}}$ & Rosemary & 47.8 & 0.353 & 649 & 0.826 & 335 & 0.63 & 37.5 & 1.647 & 44.1 & 0.330 \\
\hline Sesbania cannabina Merr. & Prickly Sesban & 60.5 & 0.858 & 988 & 1.030 & 335 & 0.63 & 47.5 & 1.142 & 44.9 & 0.298 \\
\hline Plantanus occidentalis ${ }^{\mathrm{f}}$ & East. Sycamore & 50.5 & 0.94 & 1090 & 1.208 & 335 & 0.63 & 1254 & 1.06 & 7.09 & 0.153 \\
\hline Fraxinus latifolia $^{\mathrm{f}}$ & Oregon Ash & 24.3 & 0.50 & 46.4 & 0.819 & 335 & 0.63 & 19.1 & 1.50 & 5.89 & 0.367 \\
\hline Panicum virgatum ${ }^{\mathrm{f}}$ & Switchgrass & 35.2 & 1.78 & 22,100 & 1.78 & 400 & 0.70 & 27.6 & 0.22 & $1.66 \times 10^{-9}$ & -2.182 \\
\hline Salix viminalis ${ }^{\mathrm{g}}$ & Willow & 21.3 & 0.631 & 180 & 1.06 & 335 & 0.63 & 16.8 & 1.37 & 5.35 & 0.231 \\
\hline
\end{tabular}

${ }^{\mathrm{a}}$ To simplify comparison with previously published values, preexponential parameters are multiplied by $\phi_{o}$ to the proper exponent. In all cases, $\phi_{o}=1 \mathrm{~mm}$. Values in italics are assumed because no data exists. For switchgrass the assumed values come from Waldron and Dakessian [1981]; All others are from Schwarz et al. [2010a]. Measurements of Operstein and Frydman [2000] were fitted to power law equations from figures. Values for switchgrass differ significantly from other species probably because $\alpha$ and $\beta$ are identical. $\beta$ was not measured but assumed [see Thomas and Pollen-Bankhead, 2010].

${ }^{\mathrm{b}}$ Schwarz et al. [2010a].

${ }^{\mathrm{c}}$ Waldron and Dakessian [1981].

${ }^{\mathrm{d}}$ Operstein and Frydman [2000].

${ }^{\mathrm{e}} \mathrm{Fan}$ and $\mathrm{Su}$ [2008].

${ }^{\mathrm{f}}$ Thomas and Pollen-Bankhead [2010].

${ }^{\mathrm{g}}$ Mickovski et al. [2009]. 


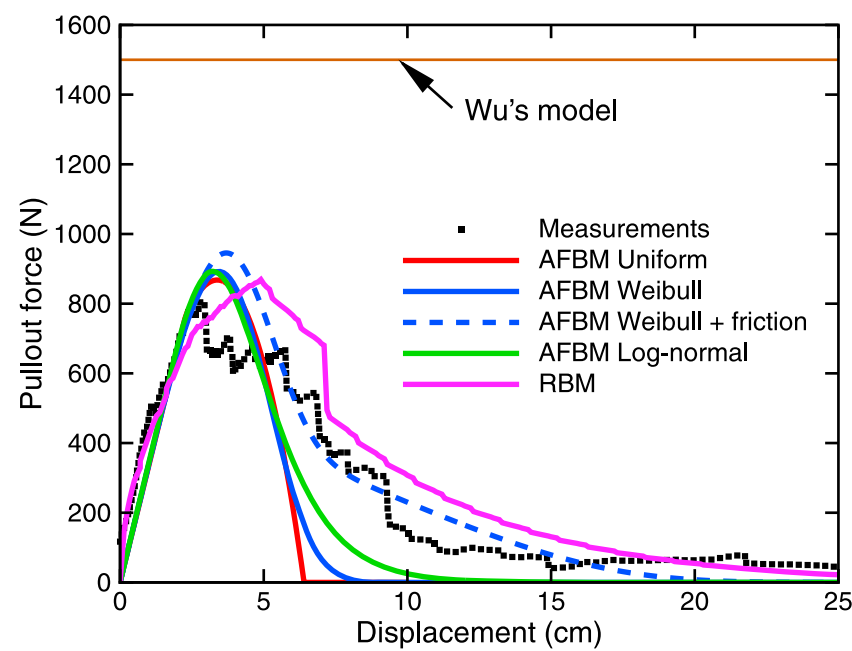

Figure 6. Comparison between measured pullout force as a function of displacement (black squares) [Schwarz et al., 2011] and the analytical fiber bundle model (AFBM) with uniform, Weibull, and lognormal diameter distributions. The value of $E_{o}$ used in the calculations is half the value mentioned by Schwarz et al. [2010] (Table 5) to account for root tortuosity. Also shown is the inclusion of root-soil friction on the AFBM pullout behavior for the Weibull distribution (dashed blue line) using a value for root-soil friction $(\tau)$ of $2000 \mathrm{~Pa}$ in accord with previous estimates [Waldron, 1977; Schwarz et al., 2011] and a root broken fraction $(\xi)$ of 0.9 (details of the root-soil friction model are given in Appendix A). Results from the numerical root bundle model (RBM) of Schwarz et al. [2010] is also shown for comparison. For comparison, the model of $\mathrm{Wu}$ et al. [1979] gives a value of about $1500 \mathrm{~N}$ for all values of displacement, almost twice as much as the maximum force measured.

power law exponent $\kappa$ that controls tensile forces in roots (equation (27)). This discrepancy is difficult to resolve: values of $\gamma$ and $L_{o}$ given by Waldron and Dakessian [1981] were not measured and thus are only approximations. Note that our model with $\gamma=1.2$ captures the decrease of root reinforcement with increasing strain as expected from the failure and slip of roots during shear and as shown in the earlier experiments of Waldron [1977].

\subsection{Shear of Willow-Reinforced Soil}

[38] Mickovski et al. [2009] measured root reinforcement of soils planted with willow trees and compared their data with several models. From their data on root distribution (Mickovski et al.'s Table 3) it is possible to compute the Weibull distribution parameters by noting that the total root cross-sectional area (CSA, given in Mickovski et al.'s Table 3) is related to the second moment of a distribution and hence to the standard deviation. From the standard deviation and the mean diameter, one can compute a unique set of shape and scale parameters for the Weibull distribution. We obtained, respectively, 1.3 and $1.4 \mathrm{~mm}$ for the 100 $\mathrm{mm}$ depth, 1.3 and $0.87 \mathrm{~mm}$ for the $250 \mathrm{~mm}$ depth, and 1.2 and $0.68 \mathrm{~mm}$ for the $400 \mathrm{~mm}$ depth. Mickovski et al. [2009] do not give data on root length. We assume all roots have the same length equal to $400 \mathrm{~mm}\left(L_{o}=400 \mathrm{~mm}\right.$ and $\left.\gamma=0\right)$. This length, which includes root length above and below the shear plane, is less than the sample holder $(590 \mathrm{~mm})$. Other parameters used for the comparison are given in Table 6 . Figure 9 shows the maximum root reinforcement at different depth for the data and several models including ours. Our model performs either as well or better than others at all depth: a composite error calculated using the square root of the sum of the squares of the percent error for each depth measurement yields an error of $12 \%$ for our model and $31 \%$ for the next best model of $W u$ et al. [1979]. Assuming a geometric average for the root threshold strength (in terms of stress, blue square in Figure 9) is equivalent to the model of Gray and Leiser [1982] and overestimates reinforcement by about a factor of 2. Using the model of Wu et al. [1979] by summing over individual roots instead of root classes (cyan squares in Figure 9) yields a good agreement with the observations, in contrast with the data of Schwarz et al. [2011] presented in section 3.1 and in Figure 6. This is likely due to the small and narrow range of root diameters in the laboratory experiments of Mickowski et al. [2009]. This narrow range of small-diameter roots effectively behaves like a bundle of identical roots and, in that case, summing the maximum strength of each root as is done by the $W u$ et al. [1979] model is not very different from the progressive failure of roots using the analytical fiber bundle model (see discussion at end of section 2.5).

[39] Contrary to the fiber bundle model of Pollen and Simon [2005], our analytical fiber bundle model does not underpredict maximum root reinforcement in these cases. A possible explanation is that our model renders better the dynamics of the shear stress tests by imposing a constant displacement rate on all roots. In contrast, the model of Pollen and Simon [2005] pulls the bundle of roots with the equivalent of a compliant puller using a load redistribution rule that does not necessarily guarantee identical displacements for all roots.

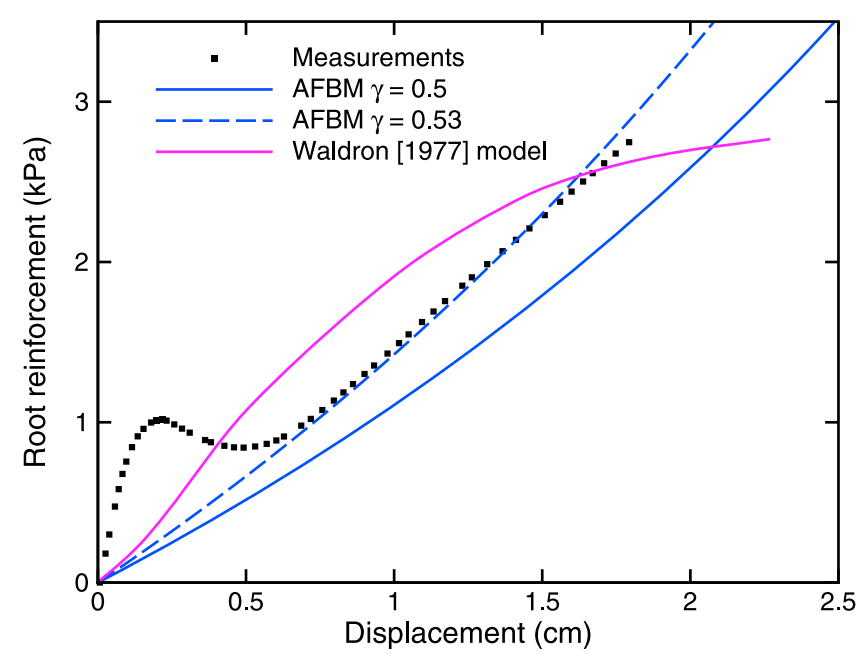

Figure 7. Comparison between measured root reinforcement in shear stress tests of a soil planted with barley as a function of displacement [Waldron and Dakessian, 1981], the analytical fiber bundle model for two values of $\gamma$, and the model of Waldron [1977]. 


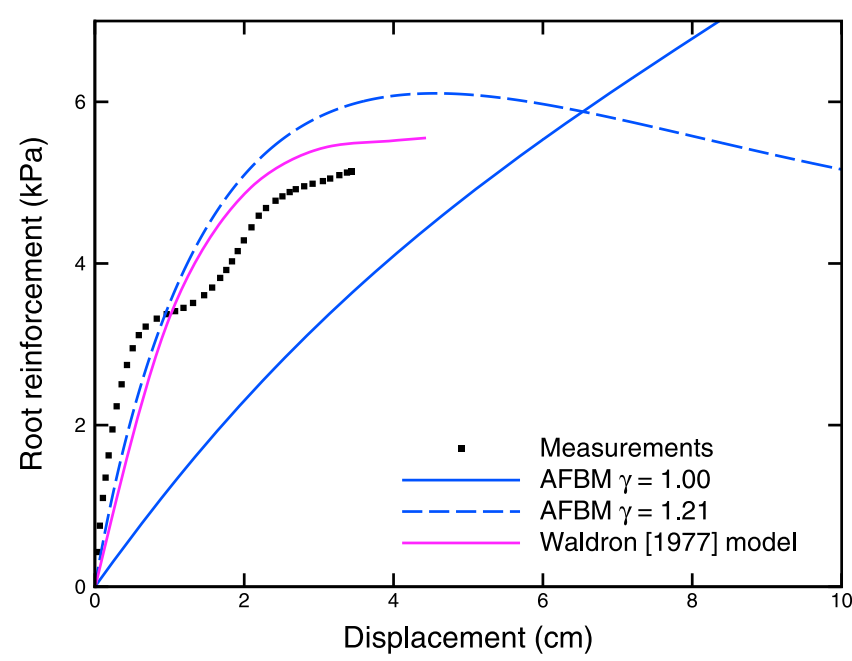

Figure 8. Comparison between measured root reinforcement in shear stress tests of a soil planted with pine as a function of displacement [Waldron and Dakessian, 1981], the analytical fiber bundle model for two values of $\gamma$, and the model of Waldron [1977].

\subsection{Shear of Root-Reinforced Colluvium}

[40] Fannin et al. [2005] performed a series of in situ shear tests on cohesionless colluvium to explore strength mobilization in shallow translational slips. In one shear test (see Figure 1, solid squares), they observed a second peak in the stress which they attributed to roots crossing the shear surface. To illustrate the independent influences of matrix and roots on the total shear strength of soil, we model the constitutive curve of Fannin et al. [2005] by isolating the effects of roots and soil by subtracting the second peak (due to roots) from the base curve (peak strength and residual strength due to matrix). The two solid lines in Figure 1 (green for the matrix, brown for the roots) show the outcome of this procedure. The stress-strain curve for the matrix component (green curve) was fitted to a fiber bundle made of elastic-plastic fibers [Raischel et al., 2006] pulled under tension which reproduces the peak strength of the matrix and the residual strength at higher displacement (see Cohen et al. [2009, Figure 12c] for details). Lacking any information on root characteristics (whether geometrical or mechanical), we fitted the data with the present analytical model, arbitrarily using 76 roots with $T_{o}=1.944 \times 10^{5} \mathrm{~Pa}$, $\alpha=0.72, E_{o}=1.06 \times 10^{6} \mathrm{~Pa}, \beta=1, L_{o}=26 \mathrm{~m}, \gamma=0.63$, and a Weibull distribution with $m_{\phi}=2.48$ and $k_{\phi}=1 \mathrm{~mm}$. Based on the sudden increase of strength prior to the second peak, we assumed that roots provided shear resistance only after about $18 \mathrm{~cm}$ of shear displacement, probably owing to tortuosity in a lightly compacted matrix (all of Fannin et al.'s [2005] experiments were performed under very low normal stress in relatively coarse material $\left(D_{50}=0.6 \mathrm{~mm}\right.$ with $10 \%$ gravel)). The sum of the fiber bundle model for the matrix and the one for the root is shown as a dashed red curve in Figure 1. Results fit data well and again emphasize that mobilization of root tensile strength cannot, in general, be added to the Mohr-Coulomb rule as an apparent cohesion term since root strength mobilization and matrix full mobilization occur at very different displacements.

\section{Discussion}

[41] The new analytical fiber bundle model describes pullout behaviors of root bundles that can be used to estimate root reinforcement in slope-stability models of rootpermeated soils. Although pullout behavior depends on five independent parameters (see equations in Table 3), practical applications require knowledge of eight quantities (from which the five independent parameters are estimated): (1) three root property exponents $\alpha, \beta, \gamma$ (or $\lambda, \beta, \gamma$ ); (2) three characteristic quantities $T_{o}, E_{o}, L_{o}$ (or $F_{o}, E_{o}, L_{o}$ ); and (3) two force (or diameter) root distribution parameters. For force these are $F_{a}, F_{b}$ (uniform), $m_{F}, k_{F}$ (Weibull), or $\mu_{F}$, $\sigma_{F}$ (lognormal).

[42] Note that since all probability density functions used in the present model are two-parameter distributions, knowledge of the mean and the standard deviation (for either the force or diameter distribution) are sufficient to recover the two parameters of the distributions indicated above under item 3 . We discuss below ranges and effects of these parameters on pullout behavior and on the maximum pullout force, and how they relate to other models.

\subsection{Range of Model Exponents}

[43] Imperfect materials such as natural or man-made fibers contain flaws that are randomly distributed and have random sizes. The number of these flaws and thus the possibility of having a large flaw increases with the size of the fiber [e.g., Curtin, 2000]. Thus, larger (length or diameter) fibers will, in general, fail at a smaller stress. For roots, most measurements of tensile (free roots) and pullout (roots in soil) strength [e.g., Commandeur and Pyles, 1991;

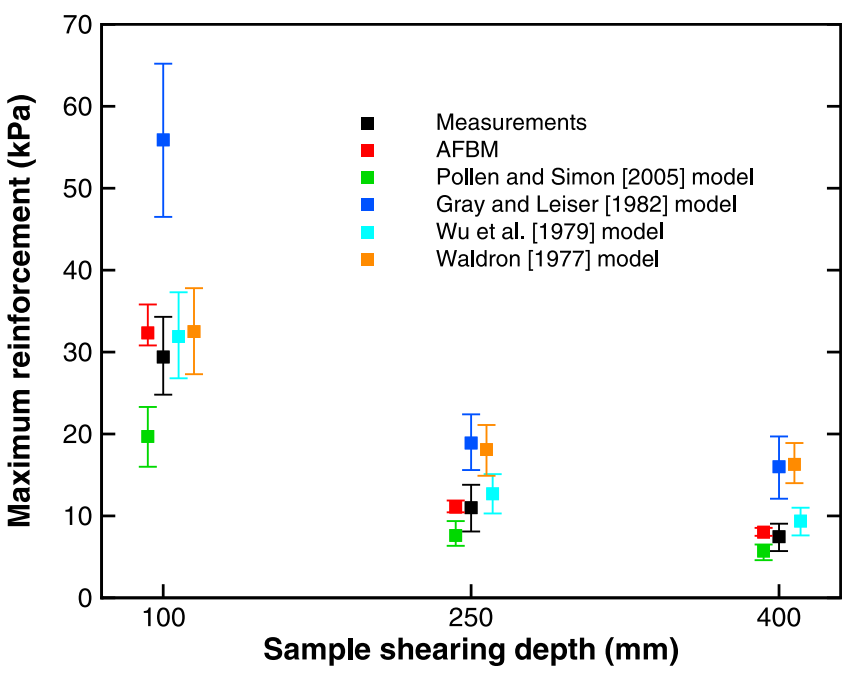

Figure 9. Comparison between measured maximum root reinforcement in shear stress tests of a soil planted with willow [Mickovski et al., 2009], the analytical fiber bundle model (AFBM), and various other models. The upper and lower bounds of the AFBM are obtained by using $L_{o}=350$ and $450 \mathrm{~mm}$. 


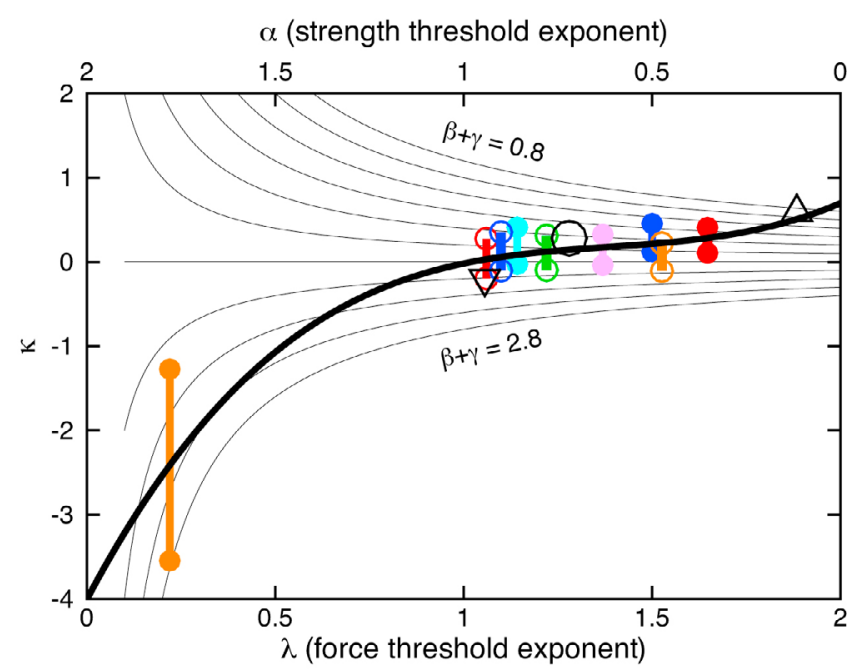

Figure 10. Data and fit of $\kappa$ as a function of $\lambda$ (or $\alpha$ ) for different species of trees and grasses. Lone symbols are for species where all exponents $(\alpha, \beta$, and $\gamma)$ are given. When $\gamma$ is not given it is assumed to range between 0.5 and 1 (vertical colored lines with open or filled circles at ends). Circle represents spruce (Picea abies L.) [Schwarz et al., 2010], upside-down triangle represents barley (Hordeum vulgare), and regular triangle represents pine (Pinus ponderosa) [Waldron and Dakessian, 1981]. Colors represent rosemary (Rosmarinus officinalis) (red, closed); alfalfa (Medicago sativa L.) (green, open); Pistacia (orange, open); Cistus [Operstein and Frydman, 2000] (blue, open); prickly sesban (Sesbania cannabina Merr.) [Fan and Su, 2008] (cyan, closed); willow (Salix viminalis cv. Tora) [Mickovski et al., 2009] (pink, closed); Alamo switchgrass (Panicum virgatum) (orange, closed); eastern sycamore (Plantanus occidentalis) (red, open); and Oregon ash (Fraxinus latifolia) [Thomas and Pollen-Bankhead, 2010] (blue, closed). The thick black line is a polynomial fit of $\kappa$ to $\lambda$ (see equation (40)). Also shown are thin black lines of constant $\beta+\gamma$ ranging from 0.8 to 2.8 with 0.2 increment.

Bischetti et al., 2005; Docker and Hubble, 2008] indicate that strength decreases with diameter and hence $\alpha>0$ in equation (19). A notable exception is the vertical pullout test of Docker and Hubble [2008] for Casuarina glauca (an Australian riparian tree) where $\alpha=-0.128$ when fitting threshold force against root diameter at the location of failure. In many pullout tests, roots are pulled upward from the soil mass and hence the normal stress increases along the root length increasing root-soil friction. Root failure may thus occur at a greater depth where the diameter is smaller than an equivalent lateral pull of a root in soil, causing an apparent decrease in the exponent $\alpha$.

[44] For most fibers, the elastic modulus decreases with diameter although measurements of some nearly flawless glass fibers show no such dependence [e.g., Otto, 1955]. Thus, $\beta \geq 0$. For roots, sparse data indicate that $\beta>0$ [e.g., Waldron and Dakessian, 1981; Operstein and Frydman, 2000; Fan and Su, 2008; Mickovski et al., 2009; Thomas and Pollen-Bankhead, 2010]. In general roots grow thicker and longer and thus $\gamma>0$. Data on roots [e.g., Commandeur and Pyles, 1991; Bischetti et al., 2005; Tosi,
2007; Hales et al., 2009; Preti and Giadrossich, 2009; Thomas and Pollen-Bankhead, 2010] also indicate that $\alpha<2$ and thus $\lambda>0$. With these constraints, $\kappa<1$. Conditions that would make $\kappa>1$ include: (1) force threshold decreasing with diameter $(\lambda<0$ or $\alpha>2)$; (2) elastic modulus increasing with diameter $(\beta<0)$; (3) short thick roots $(\gamma<0)$. The first two conditions may occur if larger roots decay, increasing flaw density with the consequences of reducing their strengths and increasing their elastic moduli. This behavior has been reported in one study [Ammann et al., 2009] and can be attributed to the loss of cellulose with aging leaving proportionally more of the stiff lignin behind [Genet et al., 2005]. Based on topological and physiological constraints, the third condition, however, is unlikely to be satisfied.

[45] Only two publications list values of all three exponents $\alpha, \beta$, and $\gamma$ : Waldron and Dakessian [1981] and Schwarz et al. [2010a]. Several publications give $\alpha$ and $\beta$, and many indicate values of $\alpha$ or $\lambda$ [see Hales et al., 2009, auxiliary material]. Using published values of the three exponents $\alpha, \beta$, and $\gamma$ (see Table 6), Figure 10 shows the range of the parameter $\kappa$ as a function of $\lambda$. When $\gamma$ is not given, it is assumed to range between 0.5 and 1 [Waldron and Dakessian, 1981]. With this condition the exponent $\kappa$ never exceeds 1 . Note that for switchgrass, $\kappa$ is negative and significantly smaller than for other species. This is because the exponent of the elastic modulus, $\beta$, is large and equal to the strength exponent, $\alpha$. $\beta$, however, was not measured but estimated from other tree roots [see Thomas and PollenBankhead, 2010]. Small values of $\kappa$ are usually caused by a high value of $\beta$.

[46] A polynomial fit between $\kappa$ and $\lambda$ (black curve, Figure 10) yields

$$
\kappa=-4.01+8.47 \lambda-5.90 \lambda^{2}+1.42 \lambda^{3},
$$

with $0<\lambda<2$. This empirical relationship eliminates the need for knowledge of both $\beta$ and $\gamma$ to compute $\kappa$ and the pullout force and thus could be useful for making estimates of root reinforcement when data on root is lacking. It should not be considered as a rule, however, and will likely evolve as more measurements of root strength, modulus, and length as a function of diameter are gathered.

\subsection{Effects of $\boldsymbol{\kappa}$ on Pullout Behavior}

[47] As discussed in the model section, the parameter $\kappa$ has a unique effect on bundle behavior because small or large roots will break first depending on the sign of $1-\kappa$. Since $\kappa$, for all practical cases, is less than 1 , small roots will break first. Smaller values of $\kappa$ increases the susceptibility of small roots to break early with deformation. Figure 11 illustrates the effect of $\kappa$ on the shape of the pullout curve for the uniform diameter distribution. In all cases, the pullout force increases linearly until displacement reaches a values of $F_{a}^{1-\kappa}$ (for $\kappa<1$ ) or $F_{b}^{1-\kappa}$ (for $\kappa \geq 1$ ) because up to that displacement no root breaks. The slope of the pullout curve is smallest for small values of $\kappa$ since a larger proportion of small roots will break first with increasing displacement, contributing no force and thus lowering the effective elastic modulus of the bundle. The pullout curves differ radically in their behavior beyond that displacement. For $\kappa=1$, all roots break simultaneously past that displacement. For $\kappa>1$, once roots start to break, the pullout 


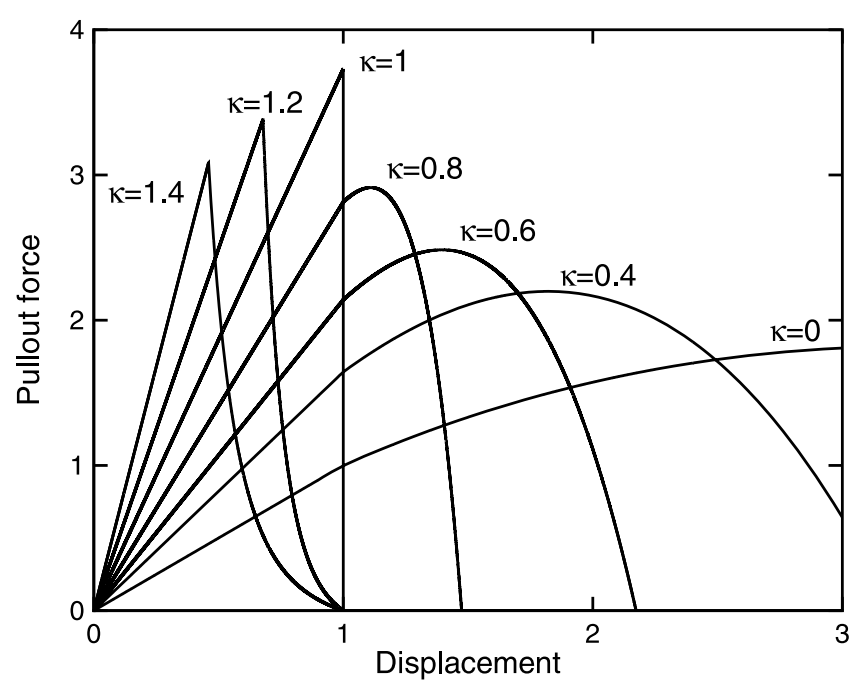

Figure 11. Dimensionless pullout force as a function of dimensionless displacement for a uniform distribution with $\phi_{a}=1, \phi_{b}=4, \lambda=1.4, F_{o}=1, W_{o}=1$, and for various values of $\kappa$ (see text above and below equation (30) for normalization of dimensional quantities). The model of $W u$ et al. [1979] implies $\kappa=1$ (see text for details).

force decreases rapidly first and then more gradually. The rapid decrease is caused by failure of large roots that hold most of the force (see Figure 3e). Smaller roots hold less force but remain intact to larger displacements. For $\kappa<1$ the opposite is true: small roots break first. As a result large roots, which remain intact and can thus hold increasingly larger forces with increasing displacement, cause the bundle pullout force to keep increasing. With increasing displacement, however, these large roots eventually rupture and the pullout force decreases. Note also that displacement at maximum force increases with decreasing $\kappa$, or equivalently with longer, less stiff (larger values of $\gamma$ or $\beta$, respectively), and weaker fibers $(\lambda \rightarrow 2)$.

[48] For distributions that span values of diameters starting from zero (e.g., Weibull, lognormal, or uniform with $\phi_{a}=0$ ), roots start breaking once displacement begins and hence the pullout curve is never linear (although it may display near-linearity initially). The effects of $\kappa$ on the pullout behavior, however, are very similar to those illustrated with the uniform distribution. Furthermore, for these distributions that span values of diameters starting from zero, the highest value of the maximum pullout force is always obtained for the curve with $\kappa=1$ when all roots break simultaneously (see Figure 3d). This special case deserves particular attention since it relates to the models of Wu et al. [1979] and of Gray and Leiser [1982]. These two cases are further discussed in the next section.

\subsection{Comparison With Average Strength Models}

[49] Both Wu's model [Wu et al., 1979] and Gray and Leiser's alternate formulation [Gray and Leiser, 1982] rely on average strength properties and assume that all roots reach their maximum strength together implying $\kappa=1$. In the case of $W u$ et al. [1979], root reinforcement, $S$, is proportional to the average of the force threshold $\langle F\rangle$ (see equation (15); we omit $n$ for simplicity assuming that the average can be taken over all roots instead of over root class size). This is equivalent to replacing equation (22) for the diameter dependence of $F$ by a constant function, i.e.,

$$
F=F_{o}=\langle F\rangle .
$$

This implies that $\lambda$, the force threshold exponent, is zero (and thus $\alpha=2$ ). Since $\kappa=1$ from the hypotheses, then $\beta+\gamma$ must be equal to exactly 2 . Since neither $\beta$ nor $\gamma$ are used in the model of $W u$ et al. [1979], the dependencies of elastic modulus $(\beta)$ and length $(\gamma)$ on diameter have no effects in the model of Wu et al. [1979].

[50] For the alternate model of Gray and Leiser [1982], the constraints on the parameters are more severe. In this case, root reinforcement is proportional to the threshold strength (a stress), $\langle T\rangle$ (see equation (17)). This is equivalent to writing

$$
T=T_{o}=\langle T\rangle,
$$

implying $\alpha=0$ and $\lambda=2$. To keep $\kappa=1$ requires $\beta+\gamma=0$. Since both parameters are practically always positive (see section 4.1), then $\beta$ and $\gamma$ must both be equal to zero. All three primary exponents, $\alpha, \beta$, and $\gamma$ are therefore null, and roots, despite having different diameters, behave mechanically and geometrically as independent of their diameters. This, and the outcome of Wu et al. [1979] model, is in direct contradiction with measurements of root properties (see section 4.1 and examples in Table 6). Wu et al. [1979] themselves measured failure loads of roots that fit $\alpha=0.2$.

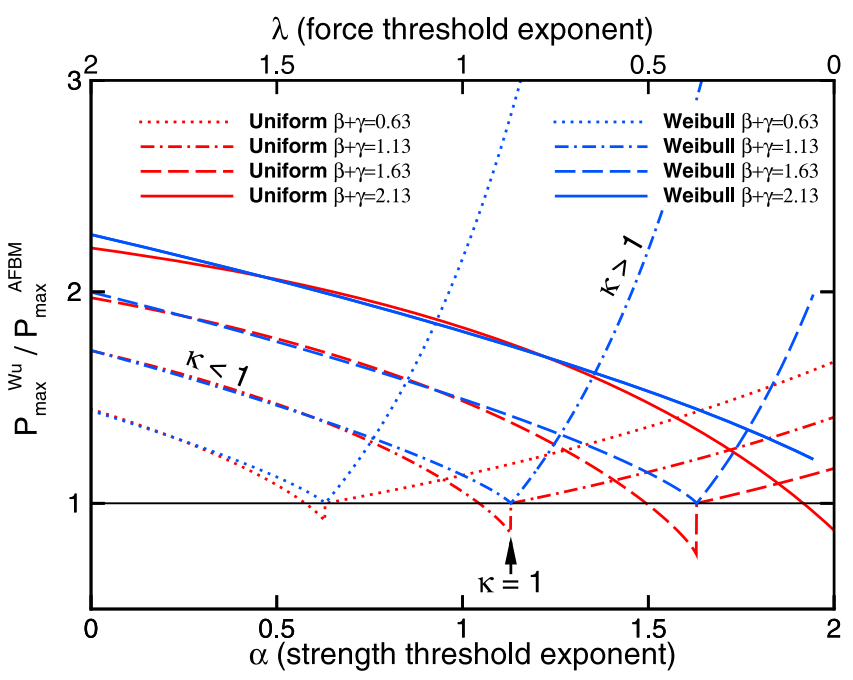

Figure 12. Ratio of maximum pullout force with $\kappa=1$ (model of $W u$ et al. [1979]) to maximum pullout force computed with the AFBM where $\kappa$ is a free parameter computed from equation (29). Results are shown as a function of $\alpha$ for various values of $\beta+\gamma$ and for the uniform and Weibull distributions. Parameters for the diameter distributions are those that fit the data of Schwarz et al. [2011] (see Table 5). Note that the value of $\kappa$ for the AFBM varies along each curve. Branches where $\kappa$ is more or less than 1 and cusps where $\kappa=1$ are indicated for the Weibull distribution for the case $\beta+\gamma=1.63$. 


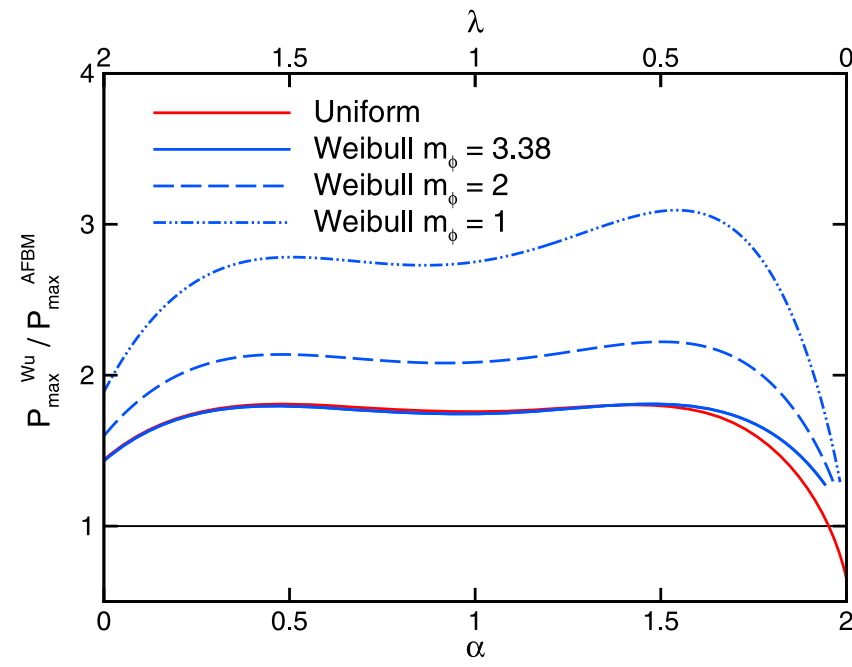

Figure 13. As in Figure 12, plot of ratio of maximum pullout force with $\kappa=1$ (Wu et al. [1979] model) to maximum pullout force for the AFBM with the added constraint that $\kappa$ and $\lambda$ are now related by the polynomial expression given in equation (40). No value of $\beta+\gamma$ is thus needed since this value is uniquely determined from the exponent $\lambda$ and the parameter $\kappa$ (see text for details). Solid curves have the same diameter distribution parameters as in Figure 12. Dashed and dash-dotted curves are with Weibull shape parameters that yield wider root-diameter distributions. With $m_{\phi}=1$ the Weibull distribution is equivalent to the exponential distribution.

Note that both Wu et al. [1979] and Gray and Leiser's [1982] models imply $\kappa=1$ but $\kappa=1$ does not imply either of these models as special combinations of nonzero $\alpha, \beta$, and $\gamma$, which imply mechanically nonidentical fibers, could lead to $\kappa=1$ (e.g., $\alpha=\beta+\gamma=1$ ).

[51] Assuming $\kappa=1$, as in the model of Wu et al. [1979], usually (but not always, see next paragraph) overestimates the maximum pullout force. To illustrate the effects of $\kappa$ we use, as a starting point, the diameter distribution of Table 5 that fits data of Schwarz et al. [2011] for spruce roots. Figure 12 shows the ratio of the maximum pullout force computed with $\kappa=1$ (i.e., model of $W u$ et al. [1979]) to the maximum pullout force computed with the actual value of $\kappa$ using the AFBM. Results are presented using $\alpha$ (the exponent of root strength threshold) instead of $\kappa$ as the independent variable because of its more common usage and easy-to-interpret physical meaning. Curves are shown for various values of $\beta+\gamma$. Picking a specific example helps understand the meaning of curves on this plot. For the Weibull distribution and $\beta+\gamma=1.13$ (dash-dotted blue curve), the curve has two branches that meet at $\alpha \sim 1.1$. The left branch is for $\kappa<1$, the right branch for $\kappa>1$. The cusp is precisely where $\kappa=1$ where the ratio of the maximum forces is, by definition, 1 . The values of $\kappa$ change along both of the branches. Since for roots $\kappa$ is usually less than 1 and $\alpha$ greater than 0 , only the left branch is of interest here. For this curve then, Wu et al. [1979] model overestimates the maximum force by 1.7 at $\alpha=0$ and is off by $14 \%$ at $\alpha=1$. This overestimation is highly dependent on the parameter $\beta+\gamma$ (see other curves in Figure 12). For example, for the Weibull distribution and $\beta+\gamma=2.13$ the model of $W u$ et al. [1979] overestimates the maximum force by a factor of 2.3 at $\alpha=0$.

[52] Results for the uniform distribution (red curves in Figure 12) generally behave like the Weibull cases: curves have two branches for $\kappa<1$ (left) and $\kappa>1$ (right) and meet at $\kappa=1$. However, the ratio of forces of the left branch drops below 1 near $\kappa=1$. This is because the lower limit of the range of diameter $\left(\phi_{a}\right)$ is greater than zero and, as shown in
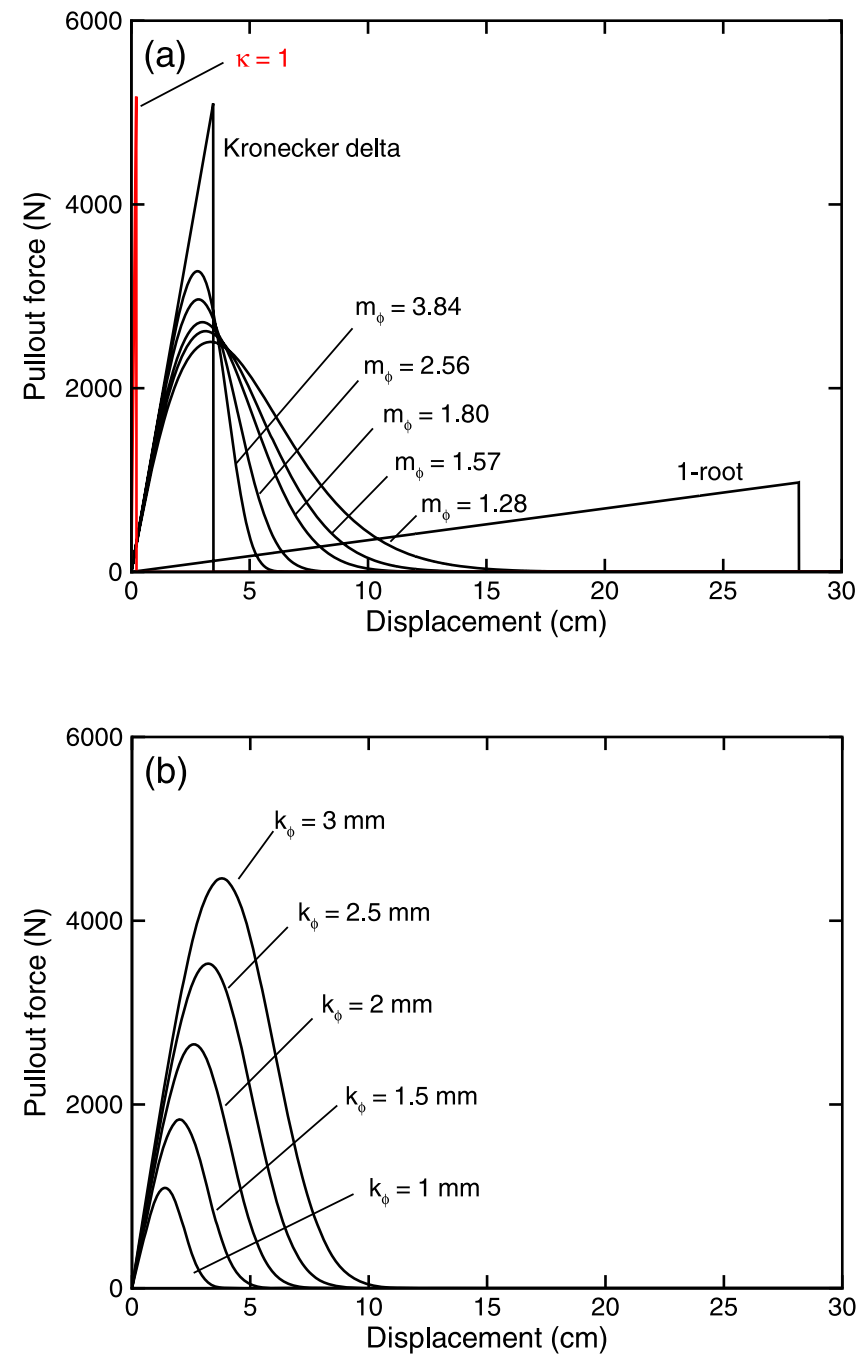

Figure 14. Effects of Weibull parameters on pullout force. The pullout force is computed for 100 roots using geometrical and mechanical parameters of Schwarz et al. [2010] (see Table 5). (a) Effect of shape parameter $m_{\phi}$ on pullout force. Values of $k_{\phi}$ were adjusted such that the average fiber diameter remains constant at $1.9 \mathrm{~mm}$. Also shown for comparison are the Kronecker delta distribution (all roots have the same diameter equal to the average diameter, see section 4.5 for details), the one-root bundle with an equivalent cross-sectional area, and the $\kappa=1$ case for $m_{\phi}=3.84$ (this case implies different values of $\alpha, \beta$, and $\gamma$ ). (b) Effect of scale parameter $k_{\phi}$. The shape parameter $m_{\phi}$ is set to 2.56 for all curves. Mean root diameter is thus different for each curve. 


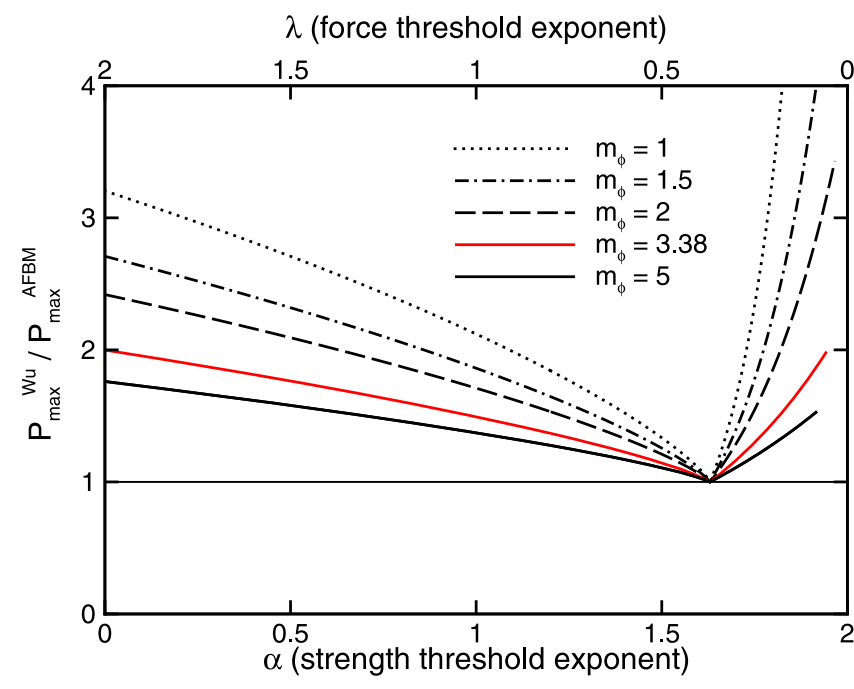

Figure 15. As in Figure 12, ratio of maximum pullout force with $\kappa=1$ (Wu et al. [1979] model) to maximum force computed with the AFBM for $\beta+\gamma=1.63$ and Weibull distributions with different values of the shape parameter $m_{\phi}$. The Weibull parameter $k_{\phi}$ is adjusted to keep the bundle mean root diameter equal to $1.9 \mathrm{~mm}$, but changing this parameter has no effect on the value of the ratio. The red curve is for the diameter distribution of Schwarz et al. [2011] (see Table 5), identical to the Weibull case $\beta+\gamma=$ 1.63 of Figure 12.

Figure 11 for $\kappa<1$, the pullout force still increases with displacement once fibers of the smallest diameters begin to break. This implies that the maximum force is greater for values of $\kappa$ just below 1 than for $\kappa=1$. This effect would disappear if $\phi_{a}$ was set to zero. Hence, results from Figure 12 indicate that the model of Wu et al. [1979] could, in some very special cases, underpredict the maximum pullout force.

[53] The parameter $\kappa$ can be estimated from $\lambda$ (or equivalently $\alpha$ ) using the fit between these two variables given by equation (40). Then, a simpler picture emerges: all $\beta+\gamma$ curves of Figure 12 collapse onto a single curve since these two parameters are no longer needed to estimate $\kappa$. Results for the uniform and Weibull are shown in Figure 13 (solid curves). They indicate that the shape of the distribution has no effect on the ratio of the maximum forces. Since $\kappa$ is always less than 1 , the ratio never approaches 1 and neither the cusp nor the right branch of the curves in Figure 12 is present. For both distributions, the AFBM indicates that $W u$ et al. [1979] model overpredicts root reinforcement by about a factor of 1.8 for much of the range of $\alpha$, the strength threshold exponent, a value within the range of previously reported overestimations of $W u$ et al. [1979] model [e.g., Pollen and Simon, 2005; Bischetti et al., 2009; Hales et al., 2009; Preti and Giadrossich, 2009].

[54] Changing the parameters of the distribution influences the ratio. To illustrate this we also show in Figure 13 curves obtained for values of the shape parameter $m_{\phi}=2$ (dashed curve) and $m_{\phi}=1$ (dash-dotted curve). $m_{\phi}=1$ is the exponential distribution so that under natural conditions $m_{\phi}$ should never be much less than 1 since that would imply an infinite amount of zero-diameter roots. These curve show that $W u$ et al. [1979] overestimation is at most 3 for $\alpha$ near 1.6 but for the most common range of $\alpha$ (less than 1) $W u$ et al. [1979] overestimation should never be more than about 2.8. This result is independent of the Weibull scale parameter $k_{\phi}$ (which is related to the mean diameter) and independent of the preexponential factors (see equations (23)-(25)) used in the calculation of the ratio since these cancel out. Specific effects of the distribution parameters are discussed in more details in the next section.

\subsection{Effect of Weibull Distribution Parameters}

[55] Schwarz et al. [2010a] emphasized the important effect of the distribution of root diameters on the maximum pullout force and its corresponding displacement. Here we present results using the Weibull distribution to illustrate these effects.

\subsubsection{Effect of Shape Parameter $m_{\phi}$}

[56] Figure 14a shows the pullout force for increasing values of the Weibull shape parameter $m_{\phi}$ (i.e., narrowing the distribution) while keeping the mean diameter of the fibers in the bundle constant (implying $k_{\phi}$ changes with changing values of $\left.m_{\phi}\right)$. With a narrower distribution, the maximum pullout force increases, the displacement at maximum force decreases, and the range of displacements over which the pullout force operates is smaller.

\subsubsection{Effect of Scale Parameter $\boldsymbol{k}_{\phi}$}

[57] Figure 14b shows the pullout force for different values of the Weibull scale parameter $k_{\phi}$ for a fixed value of $m_{\phi}$ and for bundle parameters of Schwarz et al. [2011] (Table 5) with $\kappa=0.289$. With increasing $k_{\phi}$, the bundle mean diameter increases and hence the maximum pullout force and the corresponding displacement increase. For root bundles that have $\kappa>1$ (not shown), the displacement at maximum pullout force decreases with increasing values of $k_{\phi}$.

4.4.3. Comparison With the Model of Wu et al. [1979]

[58] To complete the description of the effects of Weibull parameters on the pullout force, Figure 15 shows, as in Figure 12, the ratio of the maximum force for $\kappa=1[\mathrm{Wu}$ et al., 1979$]$ to the maximum force for actual values of $\kappa$ with the AFBM for the case $\beta+\gamma=1.63$ and for different values of $m_{\phi}$. Not surprisingly, higher values of $m_{\phi}$ give smaller ratios as this implies a narrower distribution of root diameters that tends to behave more similarly to the model of Wu et al. [1979] where all roots fail at the same time. For a given $m_{\phi}$, changing the value of $k_{\phi}$, or equivalently the mean root diameter, has almost no effect on the ratio of the maximum forces. For the left branches of the curves $(\kappa<1)$, Wu et al. [1979] model overestimates the maximum force in these particular cases by a factor of 1.7 to 3.2. These values are in agreement with results of Figure 13 where the use of a polynomial fit between $\kappa$ and $\lambda$ simplified calculations.

\subsection{Bundles of Identical Roots}

[59] A bundle of identical roots can be represented in our formulation by using the Kronecker delta probability distribution function

$$
p_{\phi}^{K}(\phi ; \bar{\phi})=\delta(\phi-\bar{\phi}),
$$



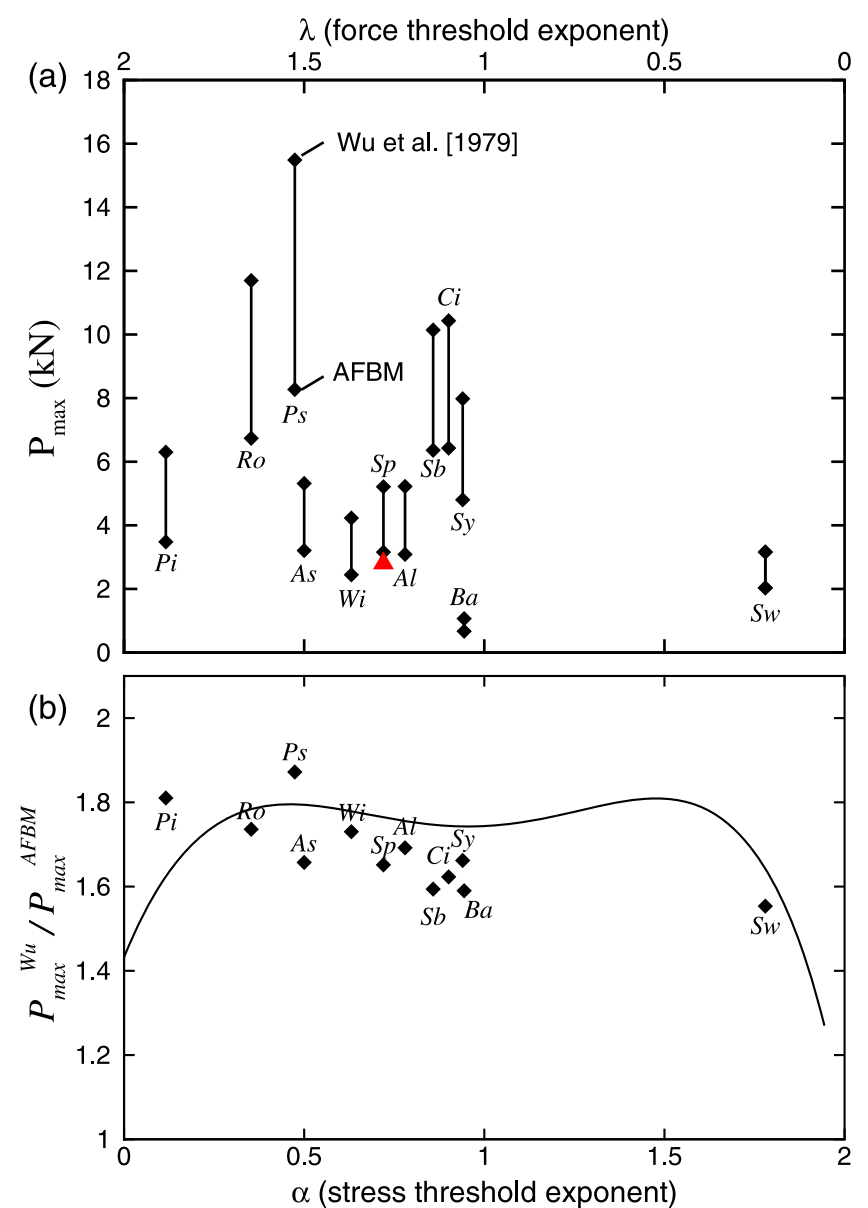

Figure 16. (a) Estimates of maximum pullout force using the analytical fiber bundle model (AFBM, lower bounds) and the Wu et al. [1979] model (upper bounds) for bundles of 100 roots as a function of $\alpha$ (stress threshold exponent) or $\lambda$ (force threshold exponent) for different plant species: $P i$, Pine; Ro, rosemary; $P s$, pistachio; $A s$, Oregon ash; $W i$, willow; $S p$, Norway spruce; $A l$, alfafa; $S b$, prickly sesban; $C i$, Cistus; $S y$, eastern sycamore; $B a$, barley; and $S w$, Alamo switchgrass. The red triangle is measurement of Schwarz et al. [2011] for Norway spruce. All bundles have identical Weibull diameter distribution (see Table 5) and geometric and mechanical characteristics of each species are given in Table 6. (b) Ratio of maximum pullout force between $W u$ et al. [1979] model and the AFBM as a function of $\alpha$ for the species (symbols) computed in Figure 16a. As in Figure 13, ratio is also computed using the polynomial fit (equation (40)) that links $\kappa$ and $\alpha$ or $\lambda$ (solid curve).

where $\bar{\phi}$ is the diameter of all the fibers in the bundle. Again using equation (22), the probability distribution function for threshold force is

$$
p_{F}^{K}(F ; \bar{F})=\left(\frac{F}{\bar{F}}\right)^{\frac{1-\lambda}{\lambda}} \delta(F-\bar{F}),
$$

where $\bar{F}$ is the threshold force. Substituting the above equation into equation (35) yields

$$
P^{K}(x)=W_{o} x \bar{F}^{\kappa},
$$

$$
P_{\max }^{K}=\bar{F}^{\kappa}
$$

and

$$
x_{\max }^{K}=\frac{\bar{F}^{1-\kappa}}{W_{o}} .
$$

For $\kappa=1$, the maximum force and corresponding displacement are identical to the $\kappa=1$ case for all distributions (see Table 4). The pullout curve for the Kronecker delta distribution is illustrated in Figure 14a together with the $\kappa=1$ case for the data of Schwarz et al. [2011]. Using fibers that have identical diameters equal to the mean diameter (but with $\kappa=0.289$, see Table 6) grossly overestimates the maximum pullout force (Kronecker delta curve, Figure 14a). Using $\kappa=1$ not only overestimates the pullout force, it also greatly underestimates the displacement at maximum force (red curve, Figure 14a). Values of $\kappa$ that approach 1 decrease drastically the displacement at maximum force as can be seen from the form of equation (47). Thus, an accurate estimate of the parameter $\kappa$ is needed to obtain the correct maximum force and corresponding displacement.

[60] Replacing a bundle of fibers with one single fiber of equivalent cross-sectional area as is done if one only knows the global root area ratio leads to an underestimation of the pullout force and an overestimation of the range of displacement the bundle can sustain (one-root curve, Figure 14a). This is in accord with the model of Waldron [1977] which states that, for an equivalent RAR, shear resistance is greater for smaller roots (see equation (9) and subsequent discussion).

\subsection{Estimates of Maximum Pullout Force}

[61] Figure 16a shows estimates of the maximum pullout force for different plant species using the AFBM and the model of $W u$ et al. [1979]. All bundles have 100 roots, identical Weibull diameter distribution (Table 5), and mechanical and geometrical root properties given in Table 6 . Also shown in Figure 16a is the only available measurement for comparison [Schwarz et al., 2011] scaled to 100 roots $(2806 \mathrm{~N})$ which matches the result of the AFBM.

[62] Figure 16b shows the ratio between values of $W u$ et al. [1979] model and the AFBM from Figure 16a (diamonds). These ratios for the different species vary between about 1.6 and 1.9, hence Wu et al. [1979] model can overestimate the pullout force (or the shear resistance $S$ ) almost $100 \%$ in these cases.

[63] Also shown in Figure 16b is the curve from Figure 13 for the Weibull distribution. This curve was obtained by assuming a polynomial fit between $\kappa$ and $\alpha$ and thus did not require information about elastic modulus $(\beta)$ or root length $(\gamma)$. In addition the preexponential factor for force threshold $\left(F_{o}\right)$ used to compute the curve was that of spruce. This curve gives a fair approximation to the actual ratio between Wu et al. [1979] model and the AFBM and thus could serve to adjust maximum estimates of root reinforcement made using Wu et al. [1979] model.

\section{Conclusions}

[64] Roots contribute to soil strength and affect slope stability whether across shear zones that cross root systems 
or along scarps where roots are pulled. An important control of root reinforcement is the tensile force held by roots during pullout. A new fiber bundle model for computing the pullout force of root bundles made of roots of different sizes and mechanical strength and stiffness is presented. In this model, roots are anchored in the ground and pulled with a rigid plate: all roots undergo identical displacements and fail progressively during pullout. The model explicitly considers the effects of tensile strength, elastic modulus, and length as a function of diameter using three power law relationships with exponents $\alpha, \beta$, and $\gamma$, respectively. We obtained analytical expressions for the pullout forces as a function of displacement for three diameter distributions: uniform, Weibull, and lognormal. For the maximum pullout force, only the uniform distribution yielded an analytical expression, the other two distribution must be solved numerically.

[65] From the analytical solutions, a parameter named $\kappa$ emerges as the main indicator of bundle pullout behavior. This parameter depends only on the three power law exponents $\alpha, \beta$, and $\gamma$. Depending on the value of $\kappa$, the bundle can behave as one of the following.

[66] 1. When $\kappa=1$, all fibers break simultaneously. This is not necessarily equivalent to a bundle of identical fibers. The model of Wu et al. [1979] falls into that category.

[67] 2. When $\kappa<1$, fibers break in increasing order of diameter. Due to constraints on the values of the three exponents, most root bundles should fall into this case.

[68] 3. When $\kappa>1$, fibers break in decreasing order of diameter. This case may happen in some unusual circumstances when old large roots become weaker and stiffer.

[69] Measurement data of root mechanics indicate that $\alpha$, the exponent of the threshold strength, is usually less than 1 except for a few species. Corresponding values of $\kappa$ are, when data are available, between 0 and 1 except for switchgrass $(\kappa<0$ ). A plot of $\kappa$ versus $\alpha$ (or $\lambda$, see section 4.1) shows that $\kappa$ can be fitted to a third-order polynomial in $\lambda$. Using this fit to compute pullout forces requires less input parameters (no need for $\beta$ and $\gamma$ ). Given the paucity of data on root elastic modulus and root length this fit could be useful.

[70] Calculations of pullout curves for species where most or all of the parameters (exponents and preexponential factors) are known (Table 6) and using the diameter distribution of Schwarz et al. [2011] (Table 5) yield significant differences in the maximum force with pistachio (Pistacia lentiscus) giving the highest resistance in tension $(16 \mathrm{kN}$ for 100 roots) and barley (Hordeum vulgare) the smallest ( $0.6 \mathrm{kN}$ also for 100 roots). For these calculations, Wu et al. [1979] model overestimates root resistance by 60 to almost $100 \%$. Based on calculations that only involve the strength exponent $\alpha$ and the parameter $\kappa$ obtained by curve fitting, the Wu et al. [1979] model will overestimate root reinforcement by at most a factor of about 3 when root distribution tend to be exponential and small roots far outnumber large ones.

[71] One key outcome of our calculations is that accurate estimates of root reinforcement requires knowledge of rootdiameter distribution. Without such knowledge and using only root-area ratio [Gray and Leiser, 1982], the overestimation of root reinforcement could be an order of magnitude higher than $\mathrm{Wu}$ et al. [1979] overestimations as has already been shown by Pollen and Simon [2005]. With knowledge of root diameter distribution and using the polynomial fit between the exponent $\kappa$ and the strength threshold exponent $\alpha$ could help correct $W u$ et al. [1979] estimates without the need for recomputing maximum forces using a fiber bundle model.

\section{Appendix A}

[72] Matrix fiber friction is a complex process cumbersome to describe, and difficult to model [e.g., Schwarz et al., 2010a]. Here we make the simplifying assumption that friction between a fiber and the matrix operates only once the fiber is broken and is proportional to the fiber surface area $A^{\prime}$ that remains in contact with the matrix times a constant frictional shear stress, $\tau^{\prime}$, namely,

$$
f_{\mu}=\tau^{\prime} A^{\prime},
$$

The area $A^{\prime}$ depends on the length of the fiber still attached to the pulling plate and is given by

$$
A^{\prime}=\pi \phi(\xi L-x), \quad 0 \leq \xi \leq 1, \quad x<\xi L,
$$

where $x$ is displacement and $\xi$ is the fraction of the fiber length $L$ that breaks and is pulled. Substituting equation (A2) into (A1) and expressing $L$ as a function of $\phi$ using equation (21) yields

$$
f_{\mu}=2 \pi \tau^{\prime}\left[\frac{\xi L_{o}}{\phi_{o}^{\gamma}} \phi^{\gamma+1}-x \phi\right] .
$$

As mentioned above, friction only operates on broken fibers except for fibers that have entirely slipped out of the soil and no longer contribute to friction (i.e., $x \geq \xi L$ ). Quantifying the total contribution of these fibers to friction requires integrating the frictional force using the diameter probability function $p_{\phi}(\phi)$, over the diameters bracketing (1) the smalldiameter fibers that have slipped out of the matrix, and (2) the larger-diameter fibers that have not yet failed, namely,

$$
P_{\mu}(x)=2 \pi N \tau^{\prime} \int_{\phi_{L}}^{\phi_{U}} f_{\mu}(x, \phi) p_{\phi}(\phi) d \phi .
$$

For $\kappa<1$,

$$
\begin{gathered}
\phi_{L}=\left(\frac{\phi_{o}^{\gamma} x}{\xi L_{o}}\right)^{1 / \gamma}, \quad \text { and } \\
\phi_{U}=\phi_{o}\left[\frac{\left(x W_{o}\right)^{1 /(1-\kappa)}}{F_{o}}\right]^{1 / \lambda} .
\end{gathered}
$$

[73] We only give here the analytical solution when $p_{\phi}(\phi)$ is a Weibull distribution with shape and scale factors $m_{\phi}$ and $k_{\phi}$, respectively. Then

$$
\begin{aligned}
P_{\mu}(x)= & \pi N \tau^{\prime} k_{\phi}\left\{\frac { k _ { \phi } ^ { \gamma } \xi L _ { o } } { \phi _ { o } ^ { \gamma } } \left[\Gamma\left(\frac{\gamma+m_{\phi}+1}{m_{\phi}}, \frac{\phi_{L}^{m_{\phi}}}{k^{m_{\phi}}}\right)\right.\right. \\
& \left.-\Gamma\left(\frac{\gamma+m_{\phi}+1}{m_{\phi}}, \frac{\phi_{U}^{m_{\phi}}}{k^{m_{\phi}}}\right)\right] \\
& \left.-x\left[\Gamma\left(\frac{m_{\phi}+1}{m_{\phi}}, \frac{\phi_{L}^{m_{\phi}}}{k^{m_{\phi}}}\right)-\Gamma\left(\frac{m_{\phi}+1}{m_{\phi}}, \frac{\phi_{U}^{m_{\phi}}}{k^{m_{\phi}}}\right)\right]\right\}
\end{aligned}
$$


where the $\Gamma$ s are the upper incomplete Gamma functions. Although cumbersome, this expression is trivial to evaluate with the help of a computer.

[74] Acknowledgments. We gratefully acknowledge the financial support of the ETH domain Center of Competence for Environmental Sustainability (CCES) through the Triggering of Rapid Mass Movement (TRAMM) project. Comments by two anonymous reviewers greatly improved the manuscript.

\section{References}

Abe, K., and R. R. Ziemer (1991), Effects of tree roots on a shear zone: Modelling reinforced shear stress, Can. J. For. Res., 21, 1012-1019.

Abernethy, B., and I. D. Rutherfurd (2001), The distribution and strength of riparian tree roots in relation to riverbank reinforcement, Hydrol. Processes, 15, 63-79.

Ammann, M., A. Böll, C. Rickli, T. Speck, and O. Holdenrieder (2009), Significance of tree root decomposition for shallow landslides, For. Snow Lanscape Res., 82, 79-94.

Beckermann, G. W., and K. L. Pickering (2009), Engineering and evaluation of hemp fibre reinforced polyupropylene composites: Micromechanics and strength prediction modelling, Composites, Part A, 40, 210-217, doi:10.1016/j.compositesa.2008.11.005.

Bischetti, G. B., E. A. Chiaradia, T. Limonato, B. Speziali, B. Vitali, P. Vullo, and A. Zocco (2005), Root strength and root area ratio of forest species in Lombardy (Northern Italy), Plant Soil, 278, 11-22.

Bischetti, G. B., E. A. Chiadaria, T. Epis, and E. Morlotti (2009), Root cohesion of forest species in the Italian Alps, Plant Soil, 342, 71-89, doi:10.1007/s11104-009-9941-0.

Charlet, J., C. Baley, C. Morvan, J. P. Jernot, M. Gomina, and J. Bréard (2007), Characteristics of Hermès flax fibres as a function of their location in the stem and properties of the derived unidirectional composites, Composites: Part A, 38, 1912-1921, doi:10.1016/j.compositesa.2007.03.006.

Cohen, D., P. Lehmann, and D. Or (2009), Fiber bundle model for multiscale modeling of hydromechanical triggering of shallow landslides, Water Resour. Res., 45, W10436, doi:10.1029/2009WR007889.

Commandeur, P. R., and M. R. Pyles (1991), Modulus of elasticity and tensile strength of Douglas-fir roots, Can. J. For. Res., 21, 48-52.

Curtin, W. A. (2000), Tensile strength of fiber-reinforced composites: III. Beyond the traditional Weibull model for fiber strengths, J. Compos. Mater., 34, 1301-1332, doi:10.1177/002199830003401503.

Curtin, W. A., and N. Takeda (1998), Tensile strength of fiber-reinforced composites: II. Application to polymer matrix composites, J. Compos. Mater., 32, 2060-2081.

Daniels, H. E. (1945), The statistical theory of the strength of bundles of threads. I, Proc. R. Soc. London, Ser. A, 183, 405-435.

Docker, B. B., and T. C. T. Hubble (2008), Quantifying root-reinforcement of river bank soils by four Australian tree species, Geomorphology, 100, 401-418.

Ekanayake, J. C., and C. J. Phillips (1999), A method for stability analysis of vegetated hillslopes: An energy approach, Can. Geotech. J., 36, 1172-1184.

Ennos, A. R. (1990), The anchorage of leek seedlings: The effect of root length and soil strength, Ann. Bot., 65, 409-416.

Fan, C. C., and C. F. Su (2008), Role of roots in the shear strength of rootreinforced soils with high moisture content, Ecol. Eng., 33, 157-166.

Fannin, R. J., A. Eliadorani, and J. M. T. Wilkinson (2005), Shear strength of cohesionless soils at low stress, Geotechnique, 55, 467-478.

Genet, M., A. Stokes, F. Salin, S. B. Mickovski, T. Fourcaud, J.-F. Dumail, and R. van Beek (2005), The influence of cellulose content on tensile strength in tree roots, Plant Soil, 278, 1-9, doi:10.1007/s11104-0058768-6.

Gray, D. H., and A. T. Leiser (1982), Biotechnical Slope Protection and Erosion Control, Van Nostrand Rheinhold, New York.

Gray, D. H., and H. Ohashi (1983), Mechanics of fiber reinforcementin sand, J. Geotech. Eng., 109, 335-353.

Gray, D. H., and R. B. Sotir (1996), Biotechnical and Soil Bioengineering Slope Stabilization: A Practical Guide for Erosion Control, WileyInterscience, New York.

Hales, T. C., C. R. Ford, T. Hwang, J. M. Vose, and L. E. Band (2009), Topographic and ecologic controls on root reinforcement, J. Geophys. Res., 114, F03013, doi:10.1029/2008JF001168.

Herrmann, H. J., and S. Roux (1990), Statistical Models for the Fracture of Disordered Media, North-Holland, Amsterdam.

Jones, B. F., and R. G. Duncan (1971), The effect of fibre diameter on the mechanical properties of graphite fibres manufactured from polyacrylonitrile and rayon, J. Mater. Sci., 6, 289-293.
Lee, K., B. Ludić, A. Magrez, J. W. Seo, G. A. D. Briggs, A. J. Kulik, and L. Forró (2007), Diameter-dependent elastic modulus supports the metastable-catalyst of growth of carbon nanotubes, Nano Lett., 7, 1598-1602, doi:10.1021/n1070502b.

Loades, K. W., A. G. Bengough, M. F. Bransby, and P. D. Hallett (2010), Planting density influence on fibrous root reinforcement of soils, Ecol. Eng., 36, 276-284, doi:10.1016/j.ecoleng.2009.02.005.

Michalowski, R. L., and A. Zhao (1996), Failure of fiber-reinforced granular soils, J. Geotech. Eng., 122, 226-234.

Mickovski, S. B., A. G. Bengough, M. F. Bransby, M. R. C. Davies, P. D. Hallet, and R. Sonnenberg (2007), Material stiffness, branching pattern and soil matric potential affect the pullout resistance of model root systems, Eur. J. Soil Sci., 58, 1471-1481.

Mickovski, S. B., P. D. Hallett, M. F. Bransby, M. C. R. Davies, R. Sonnenberg, and A. G. Bengough (2009), Mechanical reinforcement of soil by willow roots: Impacts of root properties and root failure mechanism, Soil Sci. Soc. Am. J., 73, 1276-1285, doi:10.2136/sssaj2008.0172.

Mitchell, J. K. (2005) Fundamentals of Soil Behavior, 3rd ed., John Wiley, Hoboken, N. J.

Montgomery, D. R., K. M. Schmidt, W. E. Dietrich, and J. McKean (2009), Instrumental record of debris flow initiation during natural rainfall: Implications for modeling slope stability, J. Geophys. Res., 114, F01031, doi:10.1029/2008JF001078.

O'Connell, R. A., and A. S. Yeiser (1954), Effect of crimp on mechanical properties of wet wool, Text. Res. J., 24, 629-632.

Operstein, V., and S. Frydman (2000), The influence of vegetation on soil strength, Ground Improv., 4, 81-89.

Otto, W. H. (1955), Relationship of tensile strength of glass fibers to diameter, J. Am. Ceram. Soc., 38, 122-125.

Pollen, N. (2007), Temporal and spatial variability of root reinforcement in stream banks: Accounting for soil shear strength and moisture, Catena 69, 197-205.

Pollen, N., and A. Simon (2005), Estimating the mechanical effects of riparian vegetation on stream bank stability using a fiber bundle model, Water Resour. Res., 41, W07025, doi:10.1029/2004WR003801.

Pollen, N., A. Simon, and A. Collison (2004), Advances in assessing the mechanical and hydrologic effects of riparian vegetation on stream bank stability, in Riparian Vegetation and Fluvial Geomorphology, Water Sci. Appl. Ser., vol. 8, edited by S. J. Bennett and A. Simon, pp. 125-139, AGU, Washington, D. C.

Pollen-Bankhead, N., and A. Simon (2009), Enhanced application of rootreinforcement algorithms for bank-stability modeling, Earth Surf. Processes Landforms, 34, 471-480, doi:10.1002/esp.1690.

Preti, F., and F. Giadrossich (2009), Root reinforcement and slope bioengineering stabilization by Spanish Broom (Spartium junceum L.), Hydrol. Earth Syst. Sci., 13, 1713-1726.

Raischel, F., F. Kun, and H. J. Herrmann (2006), Failure process of a bundle of plastic fibers, Phys. Rev. E, 73, 066101, doi:10.1103/PhysRevE.73. 066101.

Riestenberg, M. M., and S. Sovonick-Dunford (1983), The role of woody vegetation in stabilizing slopes in the Cincinnati area, Ohio, Geol. Sol. Am. Bull., 94, 506-518.

Rifai, S. M., and C. J. Miller (2009), Theoretical assessment of increased tensile strength of fibrous soil undergoing desiccation, J. Geotech. Geoenviron. Eng., 135, 1857-1862.

Sadek, S., S. S. Najjar, and F. Freiha (2010), Shear strength of fiber-reinforced sands, J. Geotech. Geoenviron. Eng., 136, 490-499, doi:10.1061(ASCE)GT.1943-5606.0000235.

Scanlan, C. A., and C. Hinz (2010), Using radius frequency distribution functions as a metric for quantifying root systems, Plant Soil, 332, 475-493, doi:10.1007/s11104-010-0314-5.

Schmidt, K. M., J. J. Roering, J. D. Stock, W. E. Dietrich, D. R. Montgomery, and T. Schaub (2001), The variability of root cohesion as an influence on shallow landslide susceptibility in the Oregon Coast Range, Can. Geotech. J., 38, 995-1024.

Schwarz, M., D. Cohen, and D. Or (2010a), Root-soil mechanical interactions during pullout and failure of root bundles, J. Geophys. Res., 115, F04035, doi:10.1029/2009JF001603.

Schwarz, M., F. Preti, F. Giadrossich, P. Lehmann, and D. Or (2010b), Quantifying the role of vegetation in slope stability: A case study in Tuscany (Italy), Ecol. Eng., 36, 285-291, doi:10.1016/j.ecoleng.2009. 06.014 .

Schwarz, M., D. Cohen, and D. Or (2011), Pullout tests of root analogs and natural root bundles in soil: Experiments and modeling, J. Geophys. Res., 116, F02007, doi:10.1029/2010JF001753.

Silva dos Santos, A. P., N. C. Consoli, K. S. Heineck, and M. R. Coop (2010), High-pressure isotropic compression tests on fiber-reinforced cemented sand, J. Geotech. Geoenviron. Eng., 136, 885-890, doi:10.1061/(ASCE)GT.1943-5606.0000300. 
Sornette, D. (1989), Elasticity and failure of a set of elements loaded in parallel, J. Phys., 22, L243-L250.

Thomas, R. E., and N. Pollen-Bankhead (2010), Modeling rootreinforcement with a fiber-bundle model and Monte Carlo simulation, Ecol. Eng., 36, 47-61. doi:10.1016/j.ecoleng.2009.09.008.

Tosi, M. (2007), Root tensile strength relationships and their slope stability implications of three shrubs species in the Northern Apennines (Italy), Geomorphology, 87, 268-283.

Virk, A. S., W. Hall, and J. Summerscales (2009), Multiple Data Set (MDS) weak-link scaling analysis of jute fibres, Composites, Part A, 40, 1764-1771, doi:10.1016/j.compositesa.2009.08.022.

Waldron, L. J. (1977), The shear resistance of root-permeated homogeneous and stratified soil, Soil Sci. Soc. Am. J., 41, 843-849.

Waldron, L. J., and S. Dakessian (1981), Soil reinforcement by roots: calculation of increased soil shear resistance from root properties, Soil Sci. $132,427-435$
Wu, T. H., W. P. McKinnell, and D. N. Swanston (1979), Strength of tree roots and landslides on Price of Wales Island, Alaska, Can. Geotech. J., 19-33.

Zhou, Y., D. Watts, Y. Li, and X. Cheng (1998), A case study of effect of lateral roots of Pinus yunnanensis on shallow soil reinforcement, For. Ecol. Manage., 103, 107-120.

Zornberg, J. G. (2002), Discrete framework for limit equilibrium analysis for fibre-reinforced soil, Geotechnique, 53, 593-604.

D. Cohen and D. Or, Soil and Terrestrial Environmental Physics, Institute of Terrestrial Ecosystems, ETH Zurich, Universitätstr. 16, CH-8092 Zurich, Switzerland. (denis.cohen@env.ethz.ch; dani.or@env.ethz.ch)

M. Schwarz, Swiss Federal Institute for Forest, Snow and Landscape Research, Zürcherstr. 111, CH-8903 Birmensdorf, Switzerland. (massimiliano.schwarz@wsl.ch) 